\title{
DORSAL NECK MUSCLE VIBRATION INDUCES UPWARD SHIFTS IN THE ENDPOINTS OF MEMORY-GUIDED SACCADES IN MONKEYS
}

\author{
Brian D. Corneil ${ }^{1,2}$, and Richard A. Andersen ${ }^{1}$ \\ ${ }^{1}$ Division of Biology, \\ California Institute of Technology \\ Pasadena, CA, 91126 \\ ${ }^{2}$ Departments of Physiology \& Pharmacology and Psychology \\ University of Western Ontario \\ London, ON, Canada, N6A 5K8
}

Running Head: Oculomotor effects of neck muscle vibration in monkeys

Words in abstract: 300

Number of text pages: 43

Number of figures: 12

Number of tables: 0

Correspondence should be addressed to:

Brian D. Corneil, Centre for the Brain and Mind

Robarts Research Institute, 100 Perth Drive, Dock 50, London, ON, Canada N6A 5K8 (519) 663-5777 xt 34132. FAX: (519) 663-3193. E-mail: bcorneil@uwo.ca 


\section{ABSTRACT}

Producing a movement in response to a sensory stimulus requires knowledge of the body's current configuration, and spindle organs embedded within muscles are a primary source of such kinesthetic information. Here, we sought to develop an animal model of kinesthetic illusions induced by mechanically vibrating muscles as a first step toward a mechanistic understanding of how kinesthesia is integrated into neural plans for action. We elected to examine the effects of mechanical vibration of dorsal neck muscles in head-restrained monkeys performing memory-guided saccades requiring them to look to the remembered location of a flashed target only after an imposed delay. During the delay on half of all trials, mechanical vibration (usually $1500 \mathrm{~ms}$ in duration, $200 \mu \mathrm{m}$ in amplitude, $100 \mathrm{~Hz}$ in frequency) was applied to the dorsal aspect on one side of the monkey's neck. We compared the metrics of such vibration saccades to control saccades without vibration during the delay interval. Relative to control saccades, the endpoints of vibration saccades were shifted consistently upwards, even though the variability in saccadic endpoints was unaltered. Although the stability of the eye was compromised during the delay interval of vibration trials, as evidenced by an increased incidence of upward drifts and downward microsaccades, vibration saccades displayed different metrics than control saccades, including an upwardly deviated radial direction and increased vertical amplitude. The influence of variations in the duration (500-2500 ms), amplitude (100-300 $\mu \mathrm{m})$, or frequency $(75-125 \mathrm{~Hz})$ of vibration scaled well with the presumed change in spindle activity entrained by vibration. Comparisons of the profile of these results are made to the human literature. We conclude that neck muscle vibration induces alterations in oculomotor 
performance in monkeys consistent with a central interpretation of illusory neck flexion and downward gaze deviation due to increased activation in the spindles of neck extensor muscles. 


\section{INTRODUCTION}

Generation of accurate, goal-directed actions requires knowledge of target position relative to the body's current configuration. Kinesthesia, which we define here as a sense of both position and motion, is a multisensory construct integrating visual, vestibular, somatosensory and motor information as appropriate for different body segments (see Gandevia 1996; Mergner et al. 1997; Lackner and Dizio 2000 for review). An additional predominant source of kinesthetic input is the muscle spindle organs, which relays both muscle length and velocity (see Matthews 1972 for review). One way to demonstrate the contribution of muscle spindles to kinesthesia is to mechanically vibrate muscle bellies or tendons. Provided the vibration frequency is within a physiologically relevant range, the entrainment of muscle spindle discharge to the vibration frequency results in a false signal of muscle lengthening (see Matthews 1982; Roll and Vedel 1982 for review). Numerous experiments over the past 3 decades have demonstrated that such a false signal can induce profound distortions in the body schema (e.g., Goodwin et al. 1972; Craske 1977; Lackner 1988), suggesting the proprioceptive information provided by muscle spindles plays a central role in the kinesthetic construct.

The distribution of muscle spindles throughout the body is remarkably heterogeneous (Voss 1971). From a teleological perspective, muscles with higher spindle content presumably subserve important sensory roles requiring a higher resolution. For example, the spindle content in distal finger muscles, amongst the highest in the body, likely relates to their role in active, exploratory touch. Surprisingly, a similarly high density of muscle spindles is observed in the deep muscles of the neck (Cooper and Daniel 1963; Richmond and Abrahams 1975; Richmond and Bakker 1982), and marked heterogeneities in spindle distribution are even observed within 
the same neck muscle (Richmond et al. 1999b). The microstructure and organization of neck muscle spindles appear to provide a highly resolved sense of head-on-body position. Accordingly, disruption of neck muscle afferent information induces profound disruption to not only balance and posture, but also to guided actions (Cohen 1961; Biemond and de Jong 1969; de Jong et al. 1977). Indeed, because many major exteroreceptive sense organs (e.g., eyes and ears) are mounted within a mobile head, knowledge of the position of the head relative to the body is required for many sensorimotor transformations.

Neck muscle afferent information is susceptible to alterations through mechanical vibration. Numerous human studies have documented influences of neck muscle vibration on balance, orientation or posture (Lackner and Levine 1979; Lund 1980; Karnath et al. 1994; Popov et al. 1996; Ivanenko et al. 1999; Kavounoudias et al. 1999; Lewald et al. 1999; Bove et al. 2002; Karnath et al. 2002) as well as on pointing movements (Biguer et al. 1988; Roll et al. 1991; Taylor and McCloskey 1991). The typical result is that unilateral vibration produces horizontal deviations in action or perception, consistent with the illusion of a head turn in the direction that would lengthen the muscle(s) being vibrated. Neck muscle vibration also leads to the illusory percept of motion of a light in an otherwise dark room (Biguer et al. 1988; Roll et al. 1991; Taylor and McCloskey 1991), secondary to slow-phase eye movements in the opposite direction, likely as a result of the cervico-ocular reflex (COR; Popov et al. 1999; McKenna et al. 2003). The myriad effects of neck muscle vibration on action argue that proprioceptive signals are fundamental for the sense of head-on-body position for perception and action.

The goal of this paper is to develop an animal model of some of the behavioral influences of neck muscle vibration. Development of such a model is a necessary prerequisite for a mechanistic understanding of how afferent information from neck muscles, and perhaps any 
muscle, is integrated into neural plans for action. Such a model may also provide insights into the neural mechanisms by which neck muscle vibration exerts therapeutic value in a variety of pathological conditions (e.g., Karnath et al. 1993, 2000; Schindler et al. 2002). We have elected to examine the effects of neck muscle vibration on the saccadic system of rhesus monkeys for a number of reasons. Over 40 years of research in rhesus monkeys have yielded an excellent understanding of the sensorimotor transformations underlying saccadic eye movements (Chalupa and Werner 2004), and the posterior parietal cortex in particular appears to have a primary role in integrating head position sensation for saccades (Brotchie et al. 1995; Snyder et al. 1998; Brotchie et al. 2003). Rhesus monkeys can also be trained to perform complex behavioral tasks necessary to assess the effects of neck muscle vibration. Here, we employ the endpoints of memory-guided saccades as a proxy for the influences of neck muscle vibration on the oculomotor system. Such saccades require the monkey to hold the location of a flashed visual cue in memory, and only look to the remembered location of this target after an imposed delay interval has expired. Our approach is to apply neck muscle vibration unilaterally to the dorsal neck during the delay interval on half of the trials (vibration saccades), and compare the characteristics of such vibration saccades to control saccades in which vibration was not applied during the delay interval.

Some results have been reported previously in abstract form (Corneil et al. 2003a,b).

\section{METHODS}

Experimental procedures 
Two adult male rhesus monkeys (Macaca mulatta) weighing 7.5 and $10.5 \mathrm{~kg}$ were used in these experiments following protocols approved by the Caltech Institutional Animal Care and Use Committee, and in compliance with the National Institutes of Health Guide for the Care and Use of Laboratory Animals. Both monkeys underwent an aseptic surgery during which a scleral coil was implanted subconjunctivally (Judge et al. 1980) for monitoring the horizontal and vertical rotation of the eyes in space (henceforth referred to as eye position; Fuchs and Robinson 1966; torsional eye rotations were not measured), and a head post was secured to the skull by way of a dental acrylic pedestal for immobilizing the head during the experimental sessions. Anesthesia was induced with ketamine hydrochloride and maintained with isoflurane. Antibiotics were administered pre- and post-operatively, and anti-inflammatories and analgesics were administered post-operatively. The monkeys' weights were monitored daily before and after surgery, and their general health was supervised by the university veterinarian.

Prior to the start of an experimental session, the monkeys were placed within a primate chair, and their heads restrained via the implanted head post. Within the chair, the monkeys rested on a perch with their trunk and hips facing forward. The monkeys were placed within a dark, sound-attenuated experimental chamber, in the center of magnetic fields produced by $1 \mathrm{~m}$ field coils (CNC Engineering). The monkeys faced a hemispheric array of red light emitting diodes (LEDs; each $4.7 \mathrm{~cd} / \mathrm{m}^{2}$ ) subtending about $\pm 40^{\circ}$ of the central visual field. These LEDs were arranged radially around a central LED, the fixation point (FP), which was located $50 \mathrm{~cm}$ straight ahead of the monkey. Horizontal and vertical eye positions were each recorded at 1000 Hz via a PLEXON system (Plexon Inc. Dallas, TX), and all aspects of the experimental paradigms were controlled by customized real-time Labview programs interfacing with the hardware through a PXI box (National Instruments). The monkeys were monitored throughout 
the experiment via an infrared camera and light-source, positioned behind the monkeys so that the infra-red light-source was not in their field of view.

\section{Experimental paradigms}

Monkeys were trained to perform a delayed-memory saccade task for a liquid reward. This task requires the monkey to look to the remembered location of a flashed target only when instructed (Fig. 1A). Trial onset was signaled by the removal of a background diffuse white light $\left(\sim 1.0 \mathrm{~cd} / \mathrm{m}^{2}\right)$, which was flashed between trials to prevent dark adaptation. The central FP was illuminated $250 \mathrm{~ms}$ later, and the monkeys were required to look at the FP within $1500 \mathrm{~ms}$. The monkeys then maintained fixation on the FP within a circular window of $3^{\circ}$ radius for $500-1500$ ms, and kept looking at the FP while a peripheral target was briefly flashed for $350 \mathrm{~ms}$. To receive the liquid reward, the monkeys had to look to the remembered location of the flashed cue within $1000 \mathrm{~ms}$ only after the central FP was extinguished, which constituted the 'go' signal (Fig. 1A). The interval between the offset of the cue and the go signal defined the delay interval. Once entering the window around the cue, the monkeys had to maintain fixation for a subsequent $500 \mathrm{~ms}$ before receiving a liquid reward. To prevent the monkeys from learning whether their memory-guided saccades were inaccurate, the target was not re-illuminated after the monkey looked into the target window.

Because the cue-directed saccades were performed in complete darkness and the target was not re-illuminated, there was considerable scatter and an upward shift in the endpoints of memory-guided saccades, which are typical in this type of task (Gnadt et al. 1991; White et al. 1994; Barton and Sparks 2001). To accommodate for this, the target window could be as large as $10^{\circ}$, and shifted upwards by up to $10^{\circ}$ relative to the veridical target location. 
The goal of this experiment is to compare the endpoints and metrics of memory-guided saccades generated with or without neck muscle vibration. To permit a sufficient amount of time for the effects of neck muscle vibration to accumulate, the monkeys were initially trained to generate memory-guided saccades following delay intervals that spanned up to $4000 \mathrm{~ms}$. Once proficient at this task, the monkeys were then acclimatized to neck muscle vibration delivered to the muscles of the dorsal neck. Vibration was generated by a commercially available permanent magnet shaker (model LDS-203, Ling Electronics) secured to a horizontal neck plate above the monkeys' shoulders. A contact surface consisting of a customized plexiglass hemisphere of 1.5 $\mathrm{cm}$ in diameter was placed in contact with the monkeys' dorsal neck, generally between $2-5 \mathrm{~cm}$ below the external occipital proturberance and $2-4 \mathrm{~cm}$ lateral of the dorsal midline. Contact was made on either the left or right side of the dorsal neck on alternating days. The shaker was driven by a custom-designed feedback amplifier capable of driving vibration peak-to-peak amplitudes over a range of 50 to $1000 \mu \mathrm{m}$ at frequencies ranging between 30 to $250 \mathrm{~Hz}$. However, neck muscle vibration never exceeded $300 \mu \mathrm{m}$ or $125 \mathrm{~Hz}$. Vibration duration was controlled by the experimental software, and ranged between 500 to $3500 \mathrm{~ms}$. The two monkeys acclimatized to vibration quite rapidly, and began making memory-guided saccades in the presence of vibration on the first day it was introduced.

The animals performed memory-guided saccades in which vibration was applied on half of all trials (vibration trials and vibration saccades) and not applied on the other half (control trials and control saccades). The standard parameters of vibration were $1500 \mathrm{~ms}$ in duration, 200 $\mu \mathrm{m}$ in amplitude, at a frequency of $100 \mathrm{~Hz}$. On vibration trials, vibration began $500 \mathrm{~ms}$ into the delay interval and persisted until the reward was delivered (Fig. 1A), and was therefore ongoing when the memory-guided saccade was generated. The duration of the delay interval was set to be 
equal in both control and vibration trials (i.e., the delay interval for control trials was $500 \mathrm{~ms}$ longer than the vibration duration). Within a block of trials, targets could be located at either 2 or 4 potential locations which were always placed at an eccentricity of $10^{\circ}$. When 2 potential cues were used, they were either placed at $10^{\circ}$ left and right or $10^{\circ}$ up and down. When 4 potential cue locations were used, they could either be located at the 4 cardinal locations, or at 4 oblique locations located at polar angles of $45^{\circ}, 135^{\circ}, 225^{\circ}$ or $315^{\circ}$ (with $0^{\circ}$ designating straight right). An experimental block consisted of about 30 successful trials of each unique combination of trial type (vibration vs. control) and target location. All trial combinations were presented an equal number of times within an experimental block by completing sub-blocks of 10 correct trials of each trial combination. Within each sub-block, the upcoming trial was randomly selected from the remaining trial combinations required to complete the sub-block.

Portions of the dataset consisted of three sequential experimental blocks wherein one of the vibration duration, amplitude or frequency was changed to one of three values, while the other two vibration parameters stayed at the standard levels. It was necessary to use a blocked design because we could not adjust the amplitude or frequency of vibration on-line. To avoid sequence effects, on a given day the monkey completed one of the 6 possible permutations of a low-medium-high sequence, and over 6 successive experimental blocks, each permutation was presented an equal number of times.

\section{Data Analysis}

Our approach is to compare the properties of control and vibration saccades generated within a block of trials. Figure 1B graphically depicts the parameters that will be used to establish the effects of neck muscle vibration, beginning with a comparison of the spatial 
endpoints (measure 1 in Fig. 1B) of vibration and control saccades. Across vibration and control saccades, we also compare the amplitude of the horizontal and vertical component (measure 2 in Fig. 1B), the horizontal and vertical position of the eye at the onset of the memory-guided saccade (measure 3 in Fig. 1B, taken relative to the average eye position over the interval from $100 \mathrm{~ms}$ prior to $50 \mathrm{~ms}$ after cue onset), the radial directions (measure 4 in Fig. 1B), the stability of the eye during the delay interval (measure 5 in Fig. 1B), and the saccadic reaction time (SRT) of memory-guided saccades relative to the go signal (measure 6 in Fig. 1B).

In order to group the datasets across the side of application, the horizontal component of some of these parameters (e.g., spatial endpoints, saccade amplitude) will be flipped if vibration was applied to the right side of neck. Thus, all results presented in this paper are presented as if vibration was applied to the left side of the neck.

Offline, the onset and offset of memory-guided saccades were detected by a custommade MATLAB program using a velocity criteria of $50^{\circ} / \mathrm{s}$. Position traces were first smoothed with a Savitzky-Golay filter ( $3^{\text {rd }}$ order, 11 point frame size). Instantaneous eye velocities were derived from the position traces using a 2 point differentiator, and then smoothed with a low-pass Butterworth filter $(\mathrm{fs} / \mathrm{fc}$ ratio $=17)$. All markings were checked by the experimenter and corrected if necessary. SRTs below $80 \mathrm{~ms}$ were excluded since these saccades were presumably anticipatory, and SRTs greater than $600 \mathrm{~ms}$ were excluded because of a presumed lack of alertness. Trials were also rejected if the direction of the first saccade was not within $\pm 90^{\circ}$ of the target direction. Overall, less than $1 \%$ of all trials were excluded using these criteria.

We analyzed ocular stability during the delay interval by counting the number of microsaccades, and deriving the mean drift velocity. These parameters were measured by first deriving de-saccaded velocity profiles by cutting out any points where the vectorial eye velocity 
exceeded $50 \%$ s. The velocity crossing threshold for microsaccades was identified as being greater than 3 standard deviations away from the mean velocity of this de-saccaded profile over the entire trial for at least $5 \mathrm{~ms}$. These microsaccades were then removed, and a least squares regression line fit to the remaining eye position traces. The mean drift velocity during the delay interval was taken as the slope of this regression line.

To analyze the difference between spatial endpoints, a 2D Kolmogorov-Smirnov (KS) statistic for the difference between distributions was determined (Press et al. 1992). This statistic is based on the cumulative probabilities constructed from the ranked data arrays broken down into 4 quadrants, and is particularly useful for limited numbers of data points for which the underlying probability distributions are unknown. Other conventional parametric and nonparametric analyses were applied depending on the distribution and origin of the datasets.

\section{RESULTS}

\section{Influence of neck muscle vibration on spatial endpoints of memory-guided saccades}

We first examine the influence of neck muscle vibration applied to the left side of the neck on memory-guided saccades, using standard vibration parameters (amplitude $=200 \mu \mathrm{m}$, frequency $=100 \mathrm{~Hz}$, duration of vibration $=1500 \mathrm{~ms}$ ). Figure 2 displays the results from a representative block of trials, presenting a xy-plot of the endpoints of control and vibration saccades directed to targets located $10^{\circ}$ to the right or left. In this example, vibration saccades end elevated and displaced slightly leftward compared to control saccades. A statistical test (2D KS test) confirmed that the differences in the endpoint distributions for vibration and control saccades were significant in this experimental block for both target directions $(\mathrm{P}=0.01$ to the 
right, $\mathrm{P}=0.04$ to the left). A comparative analysis of the variance of leftward control and vibration saccades in both the horizontal and vertical dimensions revealed little influence of vibration (horizontal standard deviation $(S D)=0.80^{\circ}$ for control saccades, $0.89^{\circ}$ for vibration saccades; vertical $\mathrm{SD}=1.68^{\circ}$ for control saccades, $1.66^{\circ}$ for vibration saccades) and only moderately altered to the right target (horizontal $\mathrm{SD}=1.42^{\circ}$ for control saccades, $1.14^{\circ}$ for vibration saccades; vertical $\mathrm{SD}=1.27^{\circ}$ for control saccades, $1.37^{\circ}$ for vibration saccades).

Similar trends were observed consistently in both monkeys. To analyze the population data, for each target in each experimental block we expressed the comparative difference in mean endpoints as a vector spanning from the mean endpoint of control saccades to the mean endpoint of vibration saccades (e.g., the difference vectors from Fig. 2 would both be directed up and to the left). Repeated 2-way ANOVAs of either the horizontal or vertical component of this difference vector across horizontal and vertical target component revealed that target location did not have a significant interaction on the difference vector $(\mathrm{P}>0.4$ in all cases $)$. Hence, we pooled the difference vectors across all target locations. The population of difference vectors shown in Fig. 3 demonstrates a trend toward upwardly deviated difference vectors, meaning that the endpoints of vibration saccades lay consistently higher than the endpoints of control saccades. In the vertical domain, this trend was highly significant (mean $\pm \mathrm{SD}=0.86 \pm 0.63^{\circ}$; a one-tailed ttest versus a mean of zero, $\mathrm{P}<10^{-10} ; 74$ of 149 (49.7\%) endpoint differences were significant by the $2 \mathrm{D} \mathrm{KS} \mathrm{test).} \mathrm{In} \mathrm{the} \mathrm{horizontal} \mathrm{domain,} \mathrm{a} \mathrm{trend} \mathrm{toward} \mathrm{an} \mathrm{ipsilateral} \mathrm{shift} \mathrm{toward} \mathrm{the} \mathrm{side} \mathrm{of}$ vibration was also highly significant, although the magnitude was less than in the vertical domain (mean $\pm \mathrm{SD}=-0.32 \pm 0.56^{\circ}$; a one-tailed t-test versus a mean of zero, $\mathrm{P}<10^{-5}$ ). Importantly, in spite of these systematic shifts in saccadic endpoints, neither the horizontal nor 
vertical variability of vibration saccades differed significantly from control saccades (paired ttests: $\mathrm{P}>0.2$ for both the horizontal and vertical variability).

Influence of neck muscle vibration on ocular stability during delay interval

Our hypothesis assumes that neck muscle vibration acts through altered neck muscle afferent activity, and accordingly it is plausible that vibration may induce eye movements through the cervico-ocular reflex (COR). We therefore examined the stability of the eye during the delay interval, comparing across vibration and control trials both the number of small microsaccades as well as the horizontal and vertical drift velocities of the desaccaded eye position traces.

Figures $4 \mathrm{~A}$ and $\mathrm{B}$ demonstrate examples of vertical eye position traces during individual control and vibration trials. Two features of vibration trials are apparent from these traces: an increased upward drift of the eye, and an increased incidence of downwardly directed microsaccades. Such features were observed consistently: the mean frequency of microsaccades was significantly greater in vibration trials than control trials (Fig. 4C, paired $t$-test, $\mathrm{P}<10^{-10}$ ), and the upward drift of the eye during the delay interval was significantly greater during vibration than control trials (Fig. 4D, paired $t$-test, $\mathrm{P}<10^{-6}$ ). A similar analysis of the horizontal drift velocity revealed a trend toward greater drift of the eye away from the side of vibration on vibration trials (paired $t$-test $\mathrm{P}<0.02$ ), although the accentuation of this drift was not as great as in the vertical domain. 
The observation of an augmented upward eye drift during the delay interval of vibration trials raises the concern that the differential distribution of saccadic endpoints could result simply because the monkeys generate saccades with exactly the same metrics that differ only in starting position. To investigate this, we performed three analyses. First, we compared the horizontal and vertical saccadic amplitudes of both control and vibration saccades. Figure 5 plots the differential distribution of saccadic amplitudes for both vibration and control saccades, using data from the same block as in Fig. 2. Similar to the analysis of saccade endpoints, vibration saccades had a greater vertical component than control saccades $(2 \mathrm{D} \mathrm{KS}$ test; $p=0.02$ to the right, $p=0.10$ to the left). Once again, this trend was consistent across the population. The vectors in Fig. 6 express the difference in horizontal and vertical saccade amplitudes across vibration and control saccades, and the preferential upward distribution of these vectors emphasize that vibration saccades had a significantly greater vertical component (mean $\pm \mathrm{SD}=0.98 \pm 0.67^{\circ}$; a one-tailed t-test versus a mean of zero, $\mathrm{P}<10^{-10}$ ). The small horizontal shift leftward (i.e., ipsilateral to the side of vibration) also reached significance, but again was of smaller magnitude than the vertical shift upward (mean $\pm \mathrm{SD}=-0.30 \pm 0.51^{\circ}$; a one-tailed t-test versus a mean of zero, $\mathrm{P}<10^{-6}$ ).

The observation that the vertical saccadic amplitude was slightly greater than the vertical saccadic endpoint is a curiosity, and suggested differences in eye position at the start of control and vibration saccades. We therefore performed a second analysis of starting eye position, relative to the averaged eye position during an interval spanning $100 \mathrm{~ms}$ before to $50 \mathrm{~ms}$ after cue onset. Consistent with the differences in vertical saccadic endpoint and vertical saccade amplitude, the vertical eye starting position was slightly, albeit significantly, lower prior to vibration saccades than control saccades across the population (mean $\pm \mathrm{SD}=-0.12 \pm 0.33^{\circ}$; a one-tailed t-test versus a mean of zero, $\mathrm{P}<10^{-6}$; an analogous analysis of horizontal eye starting 
positions revealed no significant differences: mean $\pm \mathrm{SD}=-0.02 \pm 0.22^{\circ}$; a one-tailed t-test versus a mean of zero, $\mathrm{P}=0.2$ ). The differential vertical eye starting positions is perhaps a bit surprising given that the accentuated upward drift of the eye during vibration trials shown in Fig. 4. Recall however that our computation of upward drift was performed on eye position traces after the removal of predominantly downwardly directed microsaccades. Apparently, the increased incidence of downwardly-directed microsaccades on vibration trials has the effect of moving the overall vertical eye position slightly downward prior to saccade onset. Regardless, the systematically downward difference in the vertical eye starting position induced by vibration also does not explain the systematic upward shift observed in the endpoints of vibration saccades.

Finally, we also compared the radial direction of vibration and control saccades, analyzing only the subset of those saccades directed toward horizontal targets. For each experimental block, we derived the mean direction of both vibration and control saccades, and from these two means derived a rotation that expresses the difference between the mean direction of vibration and control saccades for each experimental session. Positive values for this measure imply that vibration saccades had a more elevated direction than horizontal saccades. The results of this analysis are shown in Fig.7, and again confirm that vibration saccades were more elevated than control saccades (one-tailed $t$-test versus zero, $P<10^{-10}$; difference vector significant in 74/120 experimental sessions). Taken with the comparative analysis of saccade amplitudes and starting eye position, these directional results suggest that the shifts in spatial endpoints occur because saccade metrics are altered by neck muscle vibration.

We also analyzed saccade dynamics. A comparison of saccadic curvature, expressed as the maximum length of a perpendicular drawn from the true trajectory to a straight line spanning 
saccade onset and offset, and subsequently normalized to saccadic amplitude, revealed no significant differences in saccadic curvatures (paired t-test of curvature, $\mathrm{P}=0.35$ ). A comparison of peak vectorial saccade velocity revealed that vibration saccades were neither significantly faster nor slower than control saccades across the population (paired t-test of peak vectorial velocities, $\mathrm{P}=0.41$ ). Based on these comparisons, we conclude the dynamics of memory-guided saccades were not grossly affected by vibration.

\section{Influence of neck muscle vibration on saccade latencies}

We also analyzed the saccadic latencies (SRTs) of control and vibration saccades. From the representative example shown in Fig. 8A and B, the SRTs for vibration saccades were significantly longer than for control saccades (Wilcoxon rank sum test: rightward saccades, $\mathrm{P}<$ $10^{-7}$, leftward saccades, $\mathrm{P}<10^{-5}$ ). A strong, significant trend toward longer SRTs for vibration saccades was consistently observed across the population (Fig. 8C: Wilcoxon signed rank test of medians: $\mathrm{P}<10^{-10}, 80$ of 149 (54\%) of median differences were significant).

A possible concern about the endpoint differences shown in Fig. 3 is that they could have resulted if the endpoints for longer-latency vibration saccades drifted upward further, perhaps due to a speed-accuracy tradeoff. Such a phenomenon would bias the distribution of vibration saccades preferentially upward. To examine whether this could account for our results, we repeated the endpoint analysis, but restricted our analyses to latency ranges that overlapped for both control and vibration saccades. In spite of the exclusion of longer latency vibration saccades and shorter latency control saccades, the phenomenon of an upward shift for vibration saccades persisted (mean $\pm \mathrm{SD}=1.04 \pm 0.69^{\circ}$; a one-tailed t-test versus a mean of zero, $\mathrm{P}<10^{-5}$; 71 of 
$149(48 \%)$ endpoint differences were significant). Thus, the longer SRTs characteristic of vibration saccades also did not explain the differences in saccadic endpoints.

\section{Variations in parameters of vibrations}

If the oculomotor effects of neck muscle vibration are mediated through modulations in neck muscle afferent information, then these effects should vary systematically as the duration, amplitude or frequency of vibration are altered. Accordingly, we completed a series of sessions in which one of vibration duration, amplitude or frequency was varied between experimental blocks from the standard parameters $(1500 \mathrm{~ms}, 200 \mu \mathrm{m}, 100 \mathrm{~Hz})$ while the other parameters were kept constant. We will present the results of such variations in order, considering the effects of modulating vibration duration first.

VIBRATION DURATION: As expected, prolonging the duration of neck muscle vibration increased its influence on memory-guided saccades. Figure 9A summarizes the shift in spatial endpoints for the sample of data points in which the duration was varied between 500, 1500 and $2500 \mathrm{~ms}$ between different experimental blocks. From Fig. 9A, it is obvious that the spatial endpoints of vibration saccades were shifted progressively more left-up for longer vibration durations, relative to control saccades (both vertical and horizontal endpoint shifts were significant for all durations: one-tailed t-test versus zero, $\mathrm{P}<10^{-5}$ for all vertical endpoint shifts; $\mathrm{P}<0.01$ for all horizontal endpoint shifts). To analyze this data, we performed a Kruskal-Wallis non-parameteric ANOVA (KW test) across vibration duration, and used a post-hoc TukeyKramer test (TK test) to test whether the measure at the longest vibration duration was greater than at the shortest vibration duration. Assessing the vectorial magnitude of the endpoint shift 
confirmed that it increased with increasing vibration duration (Fig. 9Bi; KW test, $\mathrm{P}<10^{-4}$, TK test, $\mathrm{P}<0.001)$. Similar shifts were observed in the horizontal and vertical components of the saccadic eye movements (not shown), and the direction of vibration saccades were progressively shifted upward relative to control saccades for longer vibration durations (Fig. 9Bii; KW test, $\mathrm{P}=$ 0.01 , TK test, $\mathrm{P}<0.001)$. The comparative starting position of the eyes across control and vibration trials did not change with prolonged vibration durations ( $\mathrm{KW}$ test, $\mathrm{P}=0.26)$. These results confirm that the influence of vibration on saccade metrics increased for longer vibration durations.

We also examined endpoint variability and found, consistent with other results (Gnadt et al. 1991; White et al. 1994), that both the horizontal and vertical variability of both control and vibration saccades increased significantly for longer delay durations (KW test, $\mathrm{P}<0.0007$ for all tests, TK test, $\mathrm{P}<0.01)$. However, the comparative difference between vibration and control saccades only increased with prolonged durations in the vertical dimension, not the horizontal dimension ( $\mathrm{KW}$ test, $\mathrm{P}=0.58$ for horizontal variability differences, $\mathrm{P}<0.0001$ for vertical variability differences, TK test, $\mathrm{P}<0.05$ ). Thus, in the horizontal dimension, the increases in the variability for longer durations was the same for both vibration and control saccades, and it was only in the vertical dimension that the variability of vibration saccades increased more with longer durations than control saccades.

We also examined ocular stability during the fixation interval, assessing both the differences in the number of microsaccades and the vertical eye velocity across vibration and control trials. Although the number of microsaccades during vibration trials increased with prolonged vibration durations (Fig. 9Biii; KW test, $\mathrm{P}<10^{-5}$, TK test, $\mathrm{P}<0.001$ ), the difference in vertical eye velocity in vibration versus control saccades decreased with longer vibration 
durations (Fig. 9Biv; the effect of vibration was significant (KW test, $\mathrm{P}=0.01$, TK test between 500 and $1500 \mathrm{~ms}$ durations, $\mathrm{P}<0.05$ ). This suggests that the increased upward drift of the eye induced by vibration occurs at a more or less fixed velocity, and the increasing number of microsaccades for longer vibration durations is only a consequence of the prolonged delay interval.

VIBRATION AMPLTIUDE: Increasing the amplitude of vibration affected both the relative spatial endpoints as well as the ocular stability during the fixation interval (Fig. 10). The endpoints for vibration saccades were progressively shifted in a left-up direction for greater vibration amplitudes relative to control saccades (Fig. 10A; both vertical and horizontal endpoint shifts were significant across all amplitudes: one-tailed t-test versus zero, $\mathrm{P}<10^{-5}$ for all vertical endpoint shifts; $\mathrm{P}<0.05$ for all horizontal endpoint shifts). The vectorial magnitude of the endpoint shift increased with greater vibration amplitudes (Fig. 10Bi, KW test, $\mathrm{P}<10^{-4}$, TK test, $\mathrm{P}<0.001$ ), and as before this change was related to increasing saccade components and upwardly deflected directions (Fig. 10Bii, KW test, $\mathrm{P}=0.005$, TK test, $\mathrm{P}<0.05$ ), and not to differences in the relative starting position of the eyes across different vibration amplitudes (KW test, $\mathrm{P}=0.56$ ). In contrast to increases in vibration duration, larger vibration amplitudes had no effect on either the horizontal or vertical variability of vibration saccades (KW test, $\mathrm{P}>0.35$ ). Increasing the vibration amplitude also increased both the relative number of microsaccades compared to control saccades during the fixation interval (Fig. 10Ciii; KW test, $\mathrm{P}=0.001$, TK test, $\mathrm{P}<0.001$ ) as well as increasing the difference in the upward eye drift (Fig. 10Biv; KW test, $\mathrm{P}=0.007$, TK test, $\mathrm{P}<0.05)$. This latter effect contrasts to that which was observed with vibration duration, wherein the velocity of the upward eye drift did not change. 
VIBRATION FREQUENCY: Increasing the frequency of vibration also affected both the relative spatial endpoints as well as the ocular stability during the fixation interval (Fig. 11), and did so in a pattern similar to varying vibration amplitude. The endpoints for vibration saccades were progressively shifted in a left-up direction for greater vibration amplitudes relative to control saccades (Fig. 11A; vertical and horizontal endpoint shifts were significant across all amplitudes: one-tailed t-test versus zero, $\mathrm{P}<10^{-5}$ for all vertical endpoint shifts; $\mathrm{P}<0.003$ for all horizontal endpoint shifts). The vectorial magnitude of the endpoint shift increased with greater vibration frequencies (Fig. $11 \mathrm{Bi}, \mathrm{KW}$ test, $\mathrm{P}=0.01$, TK test, $\mathrm{P}<0.005$ ), and again this change was related to increasing saccade components and upwardly deflected saccadic directions (Fig. 11Bii, $\mathrm{KW}$ test, $\mathrm{P}=0.001$, TK test, $\mathrm{P}<0.005$ ), and not to differences in starting eye position (KW test, $\mathrm{P}=0.62$ ). Again, in contrast to changes in vibration duration, both the horizontal and vertical variability of vibration saccades were not altered by changes in vibration frequency (KW test, $\mathrm{P}>0.3$ ). Increasing the vibration frequencies also increased both the relative number of microsaccades compared to control saccades during the fixation interval (Fig. 10Ciii; KW test, P $=0.01$, TK test, $\mathrm{P}<0.005)$ as well as increasing the difference in the upward eye drift (Fig. 10Biv; KW test, $\mathrm{P}=0.002$, TK test, $\mathrm{P}<0.05)$.

\section{Low frequency vibration}

A final concern about the effects of neck muscle vibration is that they could be mediated by the vestibular organs, rather than neck muscle spindles, assuming that mechanical vibration conducts through the skull to the vestibular organs. In order to control for this possibility, we compared the influence of low-frequency $(30 \mathrm{~Hz})$ vibration that was presumably outside of the 
physiological range in which spindles indicate muscle lengthening to the standard parameters of vibration $(100 \mathrm{~Hz})$. Because we kept the amplitude and duration of vibration constant, we assumed that the mechanical transduction of any putative signal to the vestibular organs would be the same regardless of the vibration frequency. Over a number of experimental blocks, we consistently observed that the endpoint shifts in saccadic eye movements induced by neck muscle vibration at $30 \mathrm{~Hz}$ was negligible (Fig. 12A; vertical and horizontal endpoint shifts were insignificant: one-tailed t-test versus zero, $\mathrm{P}>0.35$ for both horizontal and vertical endpoint shifts), even though vibration at a frequency of $100 \mathrm{~Hz}$ applied to the same location (the experiment was done in a blocked fashion) induced the now familiar left-upward shift in saccadic endpoints (Fig. 12B; both vertical and horizontal endpoint shifts were significant: onetailed t-test versus zero, $\mathrm{P}<10^{-5}$ for both).

\section{DISCUSSION}

To our knowledge, ours is the first attempt to develop an animal model of the behavioral effects of muscle vibration of any kind. As such, it represents an important first step toward a mechanistic understanding of how such illusions are induced by muscle vibration. The main results of this study are that vibration of dorsal neck muscles in monkeys i) shifts the endpoints of memory-guided saccades upward, ii) compromises ocular stability while monkeys fixate a central LED, and iii) scales logically with variations in vibration duration, amplitude, and frequency. As will be explained below, these results are consistent with vibration during the delay interval inducing the perception of downward head and gaze motion that must be compensated for to look to the remembered location of a space-fixed target. This discussion will focus on some methodological considerations inherent to our experimental approach, consider 
evidence supporting our hypothetical mechanism, compare the pattern of results reported here to the human literature, and end with some speculation about whether this approach will be a useful tool for further neurophysiological investigations, particularly given the relatively modest influences of neck muscle vibration within a given experimental session.

\section{Methodological considerations}

Human studies of neck muscle vibration benefit from having the subject report the perceptual consequences of vibration, usually illusory motion of a fixated light, prior to beginning the experiments (e.g., Biguer et al. 1988; Roll et al. 1991; Taylor and McCloskey 1991; Karnath et al. 1993). Such a procedure optimizes the location of vibration in humans, but is impractical in animals without extensive training on reporting directions of veridical motion. The inability to establish an appropriate location of vibration prior to an experimental session likely explains much of the variability in our results across experimental sessions (e.g., Fig. 3). Such variability is a common observation across different human subjects (e.g., Biguer et al. 1988; Taylor and McCloskey 1991; Karnath et al. 1994; Han and Lennerstrand 1995; Lewald et al. 1999).

Inherent to any study of animal behavior is the difficulty in ensuring the animal's cooperation, lest the results be attributable to task performance under aversive conditions. A number of observations suggest that our monkeys were cooperating with the task in both control and vibration trials. First, the animals quickly acclimatized to neck muscle vibration, and began performing memory-guided saccades in the presence of neck muscle vibration on the first day it was introduced. Second, throughout all experimental sessions, we observed no tendency to perform control trials selectively at the expense of vibration trials; rather both monkeys 
performed both types of trials at an equally high rate. Third, there was no evidence for systematic differences in endpoint variability across control and vibration saccades, as might have been expected if vibration caused the animals to be distracted from the task at hand.

Another difficulty in our study lies in inferring the spread of vibration, given the anatomical complexity of the head-neck musculoskeletal system. The dorsal neck musculature of both monkeys is organized into four overlapping layers (Richmond et al. 2001), and it is not known how deeply surface vibration penetrates into these layers. This concern is aggravated by the plentiful endowment of muscle spindles in the deeper muscles closer to the spinal column (Cooper and Daniel 1963; Richmond and Abrahams 1975; Richmond and Bakker 1982). Although we cannot be sure whether the effects we have observed result from spindles residing in superficial or deep neck muscles, our demonstration that monkeys can serve as animal models for neck muscle vibration enable the development of implanted devices capable of delivering vibration selectively to certain muscle groups, or at the very least quantify the spread of vibration to other muscles.

Finally, one possible reason for the relatively modest influences of neck muscle vibration observed in this study is the degree of conflict between the spindle activity entrained by vibration and other sensory modalities. Presuming that vibration does not affect the vestibular end-organs (this point is considered more thoroughly in the following section), any sense of head-re-body motion induced by vibration is countered by other senses which encode the absence of head motion in space (e.g., the vestibular end-organs) or the absence of body motion (e.g., cutaneous receptors; foot pressure receptors). Human studies have documented the contribution of somatosensory modalities to postural sensations (e.g., Lackner et al. 2000), and if we assume that similar capabilities exist in the monkeys, this likely leads to the perception of a lack of body 
motion within the chair. Furthermore, the monkeys in our study are presumably aware that their heads are firmly restrained to the chair, which again likely counters the perception of head-rebody rotation.

Given these many methodological concerns, it is perhaps surprising that neck muscle vibration in monkeys leads to any behavioral effects in the first place. However, these concerns apply equally to studies of neck muscle vibration in humans, yet numerous studies have documented perceptual and behavioral effects that demonstrate an important role for neck proprioception in the elaboration of the body schema for action (Biguer et al. 1988; Roll et al. 1991; Taylor and McCloskey 1991; Karnath et al. 1994; Popov et al. 1996; Ivanenko et al. 1999; Kavounoudias et al. 1999; Lewald et al. 1999; Bove et al. 2001; Karnath et al. 2002). The development of an animal model of such effects enables more involved studies which can address many of these methodological concerns to lead to a more mechanistic understanding of the neural processing of neck muscle afferent information for action.

\section{Evidence supporting causal role for neck muscle spindles}

Numerous studies using a variety of methodologies have confirmed an important role for neck muscle afferent information in perception, postural control, and guided actions (e.g., Mergner et al. 1983, 1991, 2001; Nakamura and Bronstein 1995). However, given the uncertainty regarding the extent of neck muscle spindle activation via vibration, as well as the possible contributions of other sensory modalities, how certain are we that the effects of neck muscle vibration are mediated by altered discharge of neck muscle afferent information, and what are the alternative mechanisms? Barring direct measurements from muscle spindles or removal of other sensory modalities (e.g., via cutaneous anesthesia or vestibular lesions), we can 
only infer the contribution of these other modalities. However, considering the evidence from a diverse literature, our hypothesis of increased activity of neck muscle spindles is the most parsimonious explanation for our results.

We first consider the possibility that neck muscle vibration alters the output of vestibular end-organs. It has been known for some time that hair cells in both the vestibular end-organs are sensitive to mechanical vibrations of the skull (Young et al. 1977), and that skull vibration can lead to oculomotor effects similar to those described here (Lackner and Graybiel 1974). How possible is it that neck muscle vibration sets up pressure waves which propagate to and induce vibration of the skull? Such a question has not been completed resolved, and is still a matter of some debate (e.g., Betts et al. 2000; Karlberg et al. 2002, 2003; McKenna et al. 2003).

While we cannot definitely exclude the possibility that the results we have observed are not, at least in part, secondary to stimulation of the vestibular end-organs, a number of observations suggest a predominant role for neck muscle spindles. Anecdotal reports suggest that the perceptual and behavioral effects of neck muscle vibration are abolished by xylocaine injections into neck muscle bellies (Lund 1980), even though this would not influence any purported skull vibration. Furthermore, there were no systematic differences between control and vibration trials using vibration frequencies of $30 \mathrm{~Hz}$ (Fig. 12). A frequency of $30 \mathrm{~Hz}$ likely lies outside of the physiological range indicating considerable muscle lengthening (Roll et al. 1989), but since vibration amplitude and duration were unchanged, such vibration should have propagated to the skull. Although the study by Young and colleagues (1977) studied vibration frequencies down to only $50 \mathrm{~Hz}$, results in other species suggest that vibration frequencies of 30 $\mathrm{Hz}$ should excite at least a subset of vestibular end organs (Hudspeth 1989; ChristensenDalsgaard and Nairns 1993). 
There is also reason to suspect that neck muscle vibration may not induce skull vibrations in the first place. Recent animal and human studies (Freeman et al. 2000; Sohmer et al. 2000) demonstrate that skull vibration sets up fluid pressure waves, likely in the CSF, which can excite cochlear hair cells. Similar excitation can occur following vibration of the eye or cranial contents, even in the complete absence of skull vibration. Sohmer and colleagues (2000) also reported negligible auditory responses following neck muscle vibration, and although the location of vibration was left unspecified, this implies that pressure waves induced by neck muscle vibration are unlikely to induce skull vibration that subsequently transduced to the CSF. However, estimates of the propagation of vibration to the skull following vibration of the sternocleidomastoid muscle have yielded conflicted results (Betts et al. 2000; Bove et al. 2002), and it remains possible that the location of neck muscle vibration may be a prime determinant of the presence and magnitude of skull vibration.

These findings lead us to conclude that the increased afferent activity of neck muscle spindles, rather than vestibular end-organs, is the most likely route for the influence of neck muscle vibration, although we cannot completely discount possible vestibular influences. Information from cervical and vestibular sources converge at many sites throughout the neuraxis (e.g., Hongo et al. 1988; Kasper et al. 1988; Grüsser et al. 1990; Gdowski and McCrae 2000; Bottini et al. 2001), yet are conspicuously segregated in different areas of the posterior parietal cortex representing space in body-centered (area LIP) or world-centered coordinates (area 7a; Snyder et al. 1998). Compared to body-under-head rotation, the relatively selective activation of neck muscle afferents through localized mechanical vibration may be a useful methodology by which to study the origin, encoding and integration of head position signals within various brain areas. 
Effects of neck muscle vibration on memory-guided saccades

Assuming then that vibration alters neck muscle afferent information from neck muscles, how and why does this lead to the observed effects the oculomotor system? It seems likely that the ocular instability during the delay interval is due to the cervico-ocular reflex (COR). Neck muscle vibration in humans leads to nystagmus-like patterns of eye motion similar to what we observed here, and although the direction of eye motion in humans is predominantly horizontal, vertical and torsional components have been reported, particularly in clinical populations (Han and Lennerstrand 1995; Yagi and Ohyama 1996; Strupp et al. 1998; Popov et al. 1999; Karlberg et al. 2003). That human subjects report illusory light motion in the direction opposite to the slow phase eye movements during vibration is particularly compelling evidence that such a perception is secondary to eye motion induced via the COR (Strupp et al. 1998; Popov et al. 1999). Given that we observed upward slow phase eye movements in both monkeys (Fig. 4), it is plausible that they perceived downwardly-directed illusory motion of the FP during the delay interval. However, lacking an empirical report, this presumed percept remains speculative.

Regardless of whether the animals perceived illusory motion of the FP during the delay interval, the endpoints of memory-guided saccades were systematically shifted upward during vibration saccades. Such endpoint shifts are predominantly caused by saccades of altered metrics and are not simply a consequence of the accumulating upward drift during the delay interval (Figs. 5, 6). Further, the comparable endpoint variances across control and vibration trials suggest that the animals were generating saccades with the same precision. The upward shifts and prolonged SRTs of vibration saccades are also opposite to what would have been predicted if the downward microsaccades consequent to the COR were to interact with memory-guided 
saccades in a manner similar to that reported between VOR quickphases and antisaccades (Van Beuzekom and Van Gisbergen 2002).

Using the endpoints of memory-guided saccades as a proxy for the animals' estimates of target location, the animals apparently believe the target to be elevated relative to its veridical, space-fixed location. Our best explanation for this phenomenon is that the animals perceive that their gaze is deviated downwards during the delay interval of vibration trials, and compensate for this shift with a greater vertical component. The pattern of oculomotor responses with variations in vibration parameters suggests that the perception of downward gaze deviation accumulates during the delay interval depending on the integration of afferent input during the delay interval, as endpoint deviations were progressively larger for longer vibration durations, greater vibration amplitudes (consistent with greater penetration of vibration), and faster vibration frequencies (consistent with faster entrained spindle discharges).

This explanation leaves unanswered why altered afferent information from neck muscles should affect perceived gaze location to influence memory-guided saccades given that only retinocentric location of the remembered target relative to current eye position is theoretically required in a head-restrained preparation. However, both frontal and parietal oculomotor areas receive neck muscle afferent information (Barbas and Dubrovsky 1981a,b; Snyder et al. 1998; Brotchie et al. 2003), and the role of the superior colliculus extends beyond control of saccadic eye movements to fulfill a more general role in the control of eye-head gaze shifts (Freedman and Sparks 1997; Klier et al. 2001; Corneil et al. 2002). The pattern of neck muscle recruitment during isometric gaze shifts also changes as a function of initial head positions (Corneil et al. 2001). Our findings that the memory-guided saccades in head-restrained preparations are influenced by altered neck muscle vibration are consistent with the central nervous system 
utilizing neck muscle afferent information in the sensory-motor transformations for gaze shifts in general.

It is also unclear why SRTs were prolonged in vibration trials (Fig. 8). It is possible that the increased upward drift during the delay interval made the detection of FP offset more difficult, thereby prolonging SRTs. Alternatively, the sensory conflict between neck muscle afferent information and vestibular signals may lead to longer processing times prior to saccade generation.

\section{Comparison to human results}

To our knowledge, no one has examined the effects of neck muscle vibration specifically on memory-guided saccades in humans, although other experimental results are related. Most importantly, a number of groups have demonstrated the effects of neck muscle vibration on a variety of guided actions, including pointing (e.g., Biguer et al. 1988; Roll et al. 1991; Taylor and McCloskey 1991). The typical result is that open-loop movements are biased horizontally in the direction opposite to neck muscle vibration with variable vertical components. Furthermore, neck muscle vibration induces a predominantly horizontal COR-like movement, with the slowphase directed toward the side of vibration, although varied vertical and torsional movements have also been reported (e.g., Han and Lennerstrand 1995; Yagi and Ohyama 1996; Strupp et al. 1998; Popov et al. 1999). Thus, one apparent difference between humans and monkeys is that the effects of neck muscle vibration in humans are manifested usually in the horizontal dimension, yet in the vertical dimensions for monkeys.

We raise two possible explanations for this apparent species difference. Such differences may stem from the comparative size of the dorsal neck muscle mass in humans and monkeys. 
Dorsal neck muscle vibration likely influences at least two muscle groups in both humans and monkeys: splenius capitis and semispinalis capitis (semispinalis capitis has two compartments in monkeys: biventer cervicis and complexus; Kamibayashi and Richmond 1998; Richmond et al. 2001). In both species, splenius capitis turns the head horizontally, whereas semispinalis capitis acts to pitch the head up (Corneil et al. 2001, Vasavada et al. 2002). Thus, one possible explanation for the species difference in the horizontal versus vertical influences of neck muscle vibration in humans and monkeys is the proportional differences in the extensor muscles masses. The extensor muscles in monkeys are proportionally larger and more complex, a trend which likely serves to stabilize the rostrally-cantilevered head and neck against gravitational forces (Richmond et al. 1999a), particularly during the terrestrial mode of quadrupdeal locomotion preferred by rhesus monkeys (Napier and Napier 1985). We speculate that larger neck muscle masses are associated with a great per capita spindle content, which is consistent with the predominantly horizontal effects of neck muscle vibration in humans, and vertical effects in monkeys.

An alternative explanation of the differential effects in monkeys and humans lies in the location of neck muscle vibration. In our experiments, vibration was applied between $2-5 \mathrm{~cm}$ below the external occiput and $2-4 \mathrm{~cm}$ lateral of midline. In human experiments, experimenters typically optimize the location of neck muscle vibration for horizontal perceptions of self-motion or illusory light motion (e.g., Biguer et al. 1988; Taylor and McCloskey 1991; Karnath et al. 1993). The apparent species difference between humans and monkeys may therefore result simply from methodological differences in the location of vibration across different studies. Confirmation of a true species difference in the horizontal or vertical effects of neck muscle 
vibration will require experiments in both species which systematically map out the effects of different locations of vibration.

\section{Conclusions and future directions}

In closing, we believe we have successfully developed an animal model of the behavioral influences of neck muscle vibration. However, mechanistic study of neck muscle vibration will be hindered by the relatively small influences on the oculomotor system. In this study, standard parameters of vibration induced upward shifts in memory-guided saccades averaged about $1^{\circ}$ for $10^{\circ}$ target eccentricities (e.g., about $10 \%$ ). Head position gain fields in area LIP, which measure the modulation of an oculocentric tuning curve by head-on-body position, range around $1.6 \%$ per degree of change in head position (Snyder et al. 1998), thus the difference in delay period discharge in area LIP induced by neck muscle vibration will likely be only on the order of a few Hz. Furthermore, the existence of vertical head position gain fields has never been tested in area LIP for obvious technical reasons. As mentioned above, part of the reason for the relatively small influences of neck muscle vibration on the oculomotor system could be because of the conflicting messages issued by the vestibular system. A more fruitful approach may be to examine the influence of neck muscle vibration on open-loop pointing and reaching movements, where the degree of conflict between the vestibular and neck muscle afferent information will be lessened by the presumed changes in the position of the body under a stable head. 


\section{ACKNOWLEDGEMENTS}

This work was supported by a grant from the National Eye Institute. BDC was supported a long-term fellowship from the Human Frontier Science Program. We thank B. Gilliken and K. Pejsa for their help with animal husbandry, V. Shcherbatyuk for technical support, and C. Marks and T. Yao for administrative assistance. We thank Dr. S. Musallam for comments on an earlier version of this manuscript. 


\section{REFERENCES}

1. Barbas $\mathbf{H}$ and Dubrovsky B Central and peripheral effects of tonic vibratory stimuli to dorsal neck and extraocular muscles in the cat. Exp Neurol 74: 67-85, 1981 a.

2. Barbas H and Dubrovsky B Excitatory and inhibitory interactions of extraocular and dorsal neck muscle afferents in the cat frontal cortex. Exp Neurol 74: 51-66, $1981 \mathrm{~b}$.

3. Barton EJ and Sparks DL Saccades to remembered targets exhibit enhanced orbital position effects in monkeys. Vision Res 41: 2393-2406, 2001.

4. Betts GA, Barone M, Karlberg M, MacDougall H and Curthoys IS Neck muscle vibration alters visually-perceived roll after unilateral vestibular loss. Neuroreport 11: 2659$2662,2000$.

5. Biemond A and De Jong JM On cervical nystagmus and related disorders. Brain 92: 437$458,1969$.

6. Biguer B, Donaldson IM, Hein A and Jeannerod M Neck muscle vibration modifies the representation of visual motion and direction in man. Brain 111: 1405-1424, 1988.

7. Bottini G, Karnath HO, Vallar G, Sterzi R, Frith CD, Frackowiak RS and Paulesu E Cerebral representations for egocentric space: Functional-anatomical evidence from caloric vestibular stimulation and neck vibration. Brain 124: 1182-1196, 2001.

8. Bove M, Courtine $\mathbf{G}$ and Schieppati $\mathbf{M}$ Neck muscle vibration and spatial orientation during stepping in place in humans. J Neurophysiol 88: 2232-2241, 2002.

9. Brotchie PR, Andersen RA, Snyder LH and Goodman SJ Head position signals used by parietal neurons to encode locations of visual stimuli. Nature 375: 232-235, 1995.

10. Brotchie PR, Lee MB, Chen DY, Lourensz M, Jackson G and Bradley WG Jr Head position modulates activity in the human parietal eye fields. Neuroimage 18: 178-184, 2003. 
11. Chalupa LM and Werner JS. The Visual Neurosciences, volume 2. MIT Press, Cambridge MA.

12. Christensen-Dalsgaard $\mathbf{J}$ and Nairns PM Sound and vibration sensitivity of VIIIth nerve fibers in the frogs Leptodactylus albilabris and Rana pipiens pipiens. J Comp Physiol 172: 653-662, 1993.

13. Cohen LA Role of eye and neck proprioceptive mechanisms in body orientation and motor coordination. J Neurophysiol 24: 1:11, 1961.

14. Cooper S and Daniel PM Muscle spindles in man: their morphology in the lumbricals and the deep muscles of the neck. Brain 86: 563-586, 1963.

15. Corneil BD and Andersen RA Development of an animal model for kinesthetic illusions: neck muscle vibration alters the endpoints of memory-guided saccades in monkeys. Human Frontier Science Program $3^{r d}$ Annual Awardees meeting (Abstract) 3: 45, $2003 \mathrm{a}$.

16. Corneil BD and Andersen RA Neck muscle vibration alters the endpoints of delayed memory-guided saccades in monkeys. Neural control of Movement (Abstract) 5: B-2, 2003b.

17. Corneil BD, Olivier E and Munoz DP Neck muscle responses to stimulation of monkey superior colliculus. II. Gaze shift initiation and volitional head movements. $J$ Neurophysiol 88: 2000-2018, 2002.

18. Corneil BD, Olivier E, Richmond FJR, Loeb GE and Munoz DP Neck muscles in the rhesus monkey. II. Electromyographic patterns of activation underlying postures and movements. J Neurophysiol 86: 1729-1749, 2001.

19. Craske B Perception of impossible limb positions induced by tendon vibration. Science 196: 71-73, 1977.

20. De Jong PT, De Jong JM, Cohen B and Jongkees LB Ataxia and nystagmus induced by 
injection of local anesthetics in the neck. Ann Neurol 1: 240-246, 1977

21. Freedman EG and Sparks DL Activity of cells in the deeper layers of the superior colliculus of the rhesus monkey: evidence for a gaze displacement command. $J$ Neurophysiol 78: 1669-1690, 1997.

22. Freeman S, Sichel JY and Sohmer H Bone conduction experiments in animals - evidence for a non-osseus mechanism. Hear Res 146: 72-80, 2000.

23. Fuchs AF and Robinson DA A method for measuring horizontal and vertical eye movement chronically in the monkey. J Appl Physiol 21: 1068-1070, 1966.

24. Gandevia SC Kinesthesia: roles for afferent signals and motor commands. In: Handbook of Physiology. Exercise: Regulation and Integration of Multiple systems. Neural Control of Movement. Bethesda, MD: Am. Physiol. Soc., 1996, sect. 12, pt. I, chapt. 4, p. 128-172.

25. Gdowski GT and McCrea RA Neck proprioceptive inputs to primate vestibular nucleus neurons. Exp Brain Res 135: 511-526, 2000.

26. Gnadt JW, Bracewell RM and Andersen RA Sensorimotor transformation during eye movements to remembered visual targets. Vision Res 31: 693-715, 1991.

27. Goodwin GM, McCloskey DI, and Matthews PBC Proprioceptive illusions induced by muscle vibration: contribution by muscle spindles to perception? Science 175: 1382-1384, 1972.

28. Grüsser OJ, Pause M and Schreiter U Vestibular neurons in the parieto-insular cortex of monkeys (Macaca fascicularis): visual and neck receptor responses. J Physiol 430: 559-583, 1990.

29. Han $\mathbf{Y}$ and Lennerstrand $\mathbf{G}$ Eye movements in normal subjects induced by vibration activation of neck muscle proprioceptors. Acta Ophthalmol Scand 73: 414-416, 1995. 
30. Hongo T, Kitama $\mathbf{T}$ and Yoshida $\mathbf{K}$ Integration of vestibular and neck-afferent signals in the central cervical nucleus. Prog Brain Res 76: 155-162, 1988.

31. Hudspeth AJ Mechanoelectrical transduction by hair cells of the bullfrog's sacculus. Prog Brain Res 80: 129-135, 1989.

32. Ivanenko YP, Grasso R and Lacquaniti F Effect of gaze on postural responses to neck proprioceptive and vestibular stimulation in humans. J Physiol 519: 301-314, 1999.

33. Judge SJ, Richmond BJ and Chu FC Implantation of magnetic search coils for measurement of eye position: an improved method. Vision Res 20: 535-538, 1980.

34. Kamibayashi LK and Richmond FJR Morphometry of human neck muscles. Spine 23: 1314-1323, 1998.

35. Karlberg M, Aw ST, Black RA, Todd MJ, MacDougall HG and Halmagyi GM Vibration-induced ocular torsion and nystagmus after unilateral vestibular deafferentation. Brain 126: 956-964, 2003.

36. Karlberg M, Aw ST, Halmagyi GM and Black RA Vibration-induced shift of the subjective visual horizontal: a sign of unilateral vestibular deficit. Arch Otolaryngol Head Neck Surg 128: 21-27, 2002.

37. Karnath HO, Sievering $D$ and Fetter $M$ The interactive contribution of neck muscle proprioception and vestibular stimulation to subjective "straight ahead" orientation in man. Exp Brain Res 101: 140-146, 1994.

38. Karnath HO, Crist K and Hartje W Decerease of contralateral neglect by neck muscle vibration and spatial orientation of trunk midline. Brain 116: 383-396, 1993.

39. Karnath HO, Konczak H and Dichgans $\mathbf{J}$ Effect of prolonged neck muscle vibration on lateral head tilt in severe spasmodic torticollis. J Neurol Neurosurg Psychiatry 69: 658-660, 
2000.

40. Karnath HO, Reich E, Rorden C, Fetter M and Driver J The perception of body orientation after neck-proprioceptive stimulation. Effects of timing and visual cueing. Exp Brain Res 143: 350-358, 2002.

41. Kasper J, Schor RH and Wilson VJ Response of vestibular neurons to head rotations in vertical planes. II. Response to neck stimulation and vestibular-neck interactions. $J$ Neurophysiol 60: 1765-1778, 1988.

42. Kavounoudias A, Gihodes JC, Roll $\mathbf{R}$ and Roll JP From balance regulation to body orientation: two goals for muscle proprioceptive information processing? Exp Brain Res 124: 80-88, 1999.

43. Klier EM, Wang $\mathbf{H}$ and Crawford JD The superior colliculus encodes gaze commands in retinal coordinates. Nat Neurosci 4: 627-632, 2001.

44. Lackner JR and Dizio PA. Aspects of body self-calibration. Trends Cogn Sci 4: 279-288, 2000.

45. Lackner JR and Graybiel A Elicitation of vestibular side effects by regional vibration of the head. Aerosp Med 45: 1267-1272, 1974.

46. Lackner JR Some proprioceptive influences on the perceptual representation of body shape and orientation. Brain 111: 281-297, 1988.

47. Lackner JR, Levine MS Changes in apparent body orientation and sensory localization induced by vibration of postural muscles: vibratory myesthetic illusions. Aviat Space Environ Med 50: 346-354, 1979.

48. Lackner JR, Rabin E and DiZio P Fingertip contact suppresses the destabilizing influence of leg muscle vibration. J Neurophysiol 84: 2217-2224, 2000. 
49. Lewald J, Karnath HO and Ehrenstein WH Neck-proprioceptive influence on auditory lateralization. Exp Brain Res 125: 389-396, 1999.

50. Lund S Postural effects of neck muscle vibration in man. Experientia 36: 1398, 1980.

51. Matthews PBC Where does Sherrington's "muscular sense" originate? Muscles, joints, collary discharges? Annu Rev Neursci 5: 189-218, 1982.

52. Matthews PBC. Mammalian muscle receptors and their central actions. Arnold, London, 1972.

53. McKenna GJ, Peng GC and Zee DS Neck muscle vibration alters visually perceived rolls in normals. J Assoc Res Otolaryngol October 23, 2003; 10.1007/s10162-003-4005-2.

54. Mergner T, Huber W, and Becker W. Vestibular-neck interaction and transformation of sensory coordinates. J Vestib Res 7: 347-367, 1997.

55. Mergner T, Nardi GL, Becker W and Deecke $\mathbf{L}$ The role of canal-neck interaction for the perception of horizontal trunk and head rotation. Exp Brain Res 49: 198-208, 1983.

56. Mergner T, Sieblod C, Schweigart G and Becker W Human perception of horizontal trunk and head rotation in space during vestibular and neck stimulation. Exp Brain Res 85: 389404, 1981.

57. Mergner T, Nasios G, Maurer C and Becker W Visual object localization in space. Interaction of retinal, eye position, vestibular and neck proprioceptive information. Exp Brain Res 141: 33-51, 2001.

58. Nakamura T and Bronstein AM The perception of head and neck angular displacement in normal and labyrinthine-defective subjects: A quantitative study using the 'remembered saccade’ technique. Brain 118: 1157-1168, 1995.

59. Napier JR, Napier PH. The Natural History of Primates. The MIT Press, Cambridge, MA, 
1985.

60. Popov K, Lekhel H, Bronstein A and Gretsy M Postural responses to vibration of neck muscles in patients with unilateral vestibular lesions. Neurosci Lett 214: 202-204, 1996.

61. Popov KE, Lekhel H, Faldon M, Bronstein AM and Gretsy MA Visual and oculomotor responses induced by neck vibration in normal subjects and labyrinthine-defective patients. Exp Brain Res 128: 343-352, 1999.

62. Press WH, Flannery BP, Teukolsky SA and Vettering WT Numerical Recipes in C. 2nd edition. Cambridge University Press, Cambridge MA, 1992.

63. Richmond FJR and Abrahams VC Morphology and distribution of muscle spindles in dorsal muscles of the cat neck. J Neurophysiol 38: 1322-1329, 1975

64. Richmond FJR and Bakker DA Anatomical organization and sensory receptor content of soft tissues surrounding upper cervical vertebrae in the cat. J Neurophysiol 48: 49-61, 1982.

65. Richmond FJR, Corneil BD and Singh K Animal models of motor systems: cautionary tales from studies of head movement. Prog Brain Res 123: 411-416, 1999a.

66. Richmond FJR, Singh $\mathbf{K}$ and Corneil BD Marked non-uniformity of fiber-type composition in the primate suboccipital muscle obliquus capitis inferior. Exp Brain Res 125: 14-18, $1999 b$.

67. Richmond FJR, Singh K and Corneil BD Neck muscles in the rhesus monkey. I. Muscle morphometry and histochemistry. J Neurophysiol 86: 1717-1728, 2001.

68. Roll JP and Vedel JP Kinaesthetic role of muscle afferents in man, studied by tendon vibration and microneurography. Exp Brain Res 47: 177-190, 1982.

69. Roll JP, Vedel JP and Ribot E Alteration of proprioceptive messages induced by tendon vibration in man: a microneurographic study. Exp Brain Res 76: 213-222, 1989. 
70. Roll R, Velay JL and Roll JP Eye and neck proprioceptive messages contribute to the spatial coding of retinal input in visually oriented activities. Exp Brain Res 85: 423-431, 1991.

71. Schindler I, Kerkhoff G, Karnath HO, Keller I and Goldenberg G Neck muscle vibration induces lasting recovery in spatial neglect. J Neurol Neurosurg Psychiatry 73: 412-419, 2002.

72. Snyder LH, Grieve KL, Brotchie PR and Andersen RA Separate body- and worldreferenced representations of visual space in parietal cortex. Nature 394: 887-891, 1998.

73. Sohmer H, Freeman S, Geal-Dor M, Adelman C and Savion I Bone conduction experiments in humans - a fluid pathway from bone to ear. Hear Res 146: 81-88, 2000.

74. Strupp M, Arbusow V, Dieterich M, Sautier W and Brandt T Perceptual and oculomotor effects of neck muscle vibration in vestibular neuritis. Ipsilateral somatosensory substitution of vestibular function. Brain 121: 677-685, 1998.

75. Taylor JL and McCloskey DI Illusions of head and visual target displacement induced by vibration of neck muscles. Brain 114: 755-759, 1991.

76. Van Beuzekom AD and Van Gisbergen JA Interaction between visual and vestibular signals for the control of rapid eye movements. J Neurophysiol 88: 306-322, 2002.

77. Vasavada AN, Peterson BW and Delp SL Three-dimensional spatial tuning of neck muscle activation in humans. Exp Brain Res 147: 437-448, 2002.

78. Voss $\mathbf{H}$ Tabulation of the absolute and relative muscular spindle numbers in human skeletal musculature [in German]. Anat Anz 129: 562-572, 1971.

79. White JM, Sparks DL and Stanford TR Saccades to remembered target locations: an analysis of systematic and variable errors. Vision Res 34: 79-92, 1994. 
80. Yagi T and Ohyama Y Three-dimensional analysis of nystagmus induced by neck vibration. Acta Otolaryngol 116: 167-169, 1996.

81. Young ED, Fernandez $\mathbf{C}$ and Goldberg JM Responses of squirrel monkey vestibular neurons to audio-frequency sound and head vibration. Acta Otolaryngol 84: 352-360, 1977. 


\section{FIGURE LEGENDS}

FIG. 1. The memory-guided saccade task. $A$. To successfully complete the task, the monkey must look to the remembered location of the flashed target $(T)$ only after the fixation point $(F P)$ is extinguished (the "Go" signal). The delay interval spans from the offset of the flashed cue to the go signal. On vibration trials, vibration ( Vib) begins $500 \mathrm{~ms}$ after the start of the delay interval. $B$. Depiction of the saccade parameters which will be compared across vibration and control saccades. We have exaggerated measure 3 , the starting position of the eye relative to the FP, to improve its depiction.

FIG. 2. Spatial endpoints of control (blue squares) and vibration (red circles) saccades to targets located either $10^{\circ}$ to the right or left for one experimental block. Solid symbol denotes the mean endpoint, and solid ellipses circumscribe 1 standard deviation of the mean.

FIG. 3. Vector plots of the differences in spatial endpoints for control and vibration saccades for the population, pooling the vectors (thin, grey lines) across all target locations. Each vector expresses the difference between the mean control saccade endpoint and the mean vibration saccade endpoint for one target location from one experimental block. For example, a left-up line implies that the mean endpoint of vibration saccades lay left-up compared to the mean endpoint of control saccades. Thick, solid line denotes the mean vector across the entire population. The number in the bottom right denotes the number of vectors.

FIG. 4. A, B. Comparison of stability of vertical eye position during the delay interval of control trials $(A)$ and vibration trials $(B)$. Note the increased upward drift and incidence of downwardlydirected microsaccades during vibration trials. $C, D$. Comparison of mean number of 
microsaccades $(C)$ or vertical drift velocity of desaccaded eye-position trace $(D)$ across control and vibration trials. Each empty square denotes the mean observation from one experimental block. Diagonal line denotes the line of unity.

FIG. 5. Horizontal and vertical saccade amplitudes for control and vibration saccades. Same format and data from same experimental session as Fig. 2.

FIG. 6. Vector plots of the differences in saccade amplitude for control and vibration saccades. Same format as Fig. 3.

FIG 7. Rotational vector plot expressing the differences in saccade direction in control and vibration saccades for the sample population plotted on a polar plot. Each vector (thin, grey line) expresses the difference in the mean direction of vibration saccades compared to the mean direction of control saccades from one experimental block, pooled across target locations. Vectors with a positive, upward angle imply that the mean direction of vibration saccades were elevated compared to control saccades. Thick black line denotes the mean rotational vector.

FIG. 8. A,B. Histograms of saccadic reaction times (SRTs) for control (upward, empty histograms) and vibration saccades (downward, filled histograms) from a single experimental session to targets located $10^{\circ}$ to the left $(A)$ or right $(B)$. C. Comparison of SRTs for vibration and control saccades across the population, pooled across all target locations and experimental blocks. Each square compares the median SRT across control and vibration trials for a single target location in one experimental block. Diagonal dashed line denotes the line of unity.

FIG. 9. Variations in vibration duration. $A$. Vector plots expressing differences in spatial endpoints for experimental blocks using vibration durations of 500, 1500 or $2500 \mathrm{~ms}$. Each plot 
uses the same format as Fig. 2. B. Comparison of differences in various saccade parameters across vibration and control saccades for different vibration durations. Each thin grey line traces the values from one sequence of three experimental blocks in which vibration duration was varied pseudorandomly amongst the three values. Thick dark lines denote the mean values. Error bars denote standard error of the mean. Positive values imply that the given measure was greater for vibration saccades, with positive values in $i v$ denoting greater upward vertical velocities.

FIG. 10. Variations in vibration amplitudes between values of 100, 200 and $300 \mu \mathrm{m}$. Same format as Fig. 9.

FIG. 11. Variations in vibration frequencies between values of 75, 100 and $125 \mathrm{~Hz}$. Same format as Fig. 9.

FIG. 12. Vector plots of endpoint shifts with vibration frequency set to $30 \mathrm{~Hz}(A)$ or $100 \mathrm{~Hz}(B)$. Each subplot uses the same format as Fig. 2. 

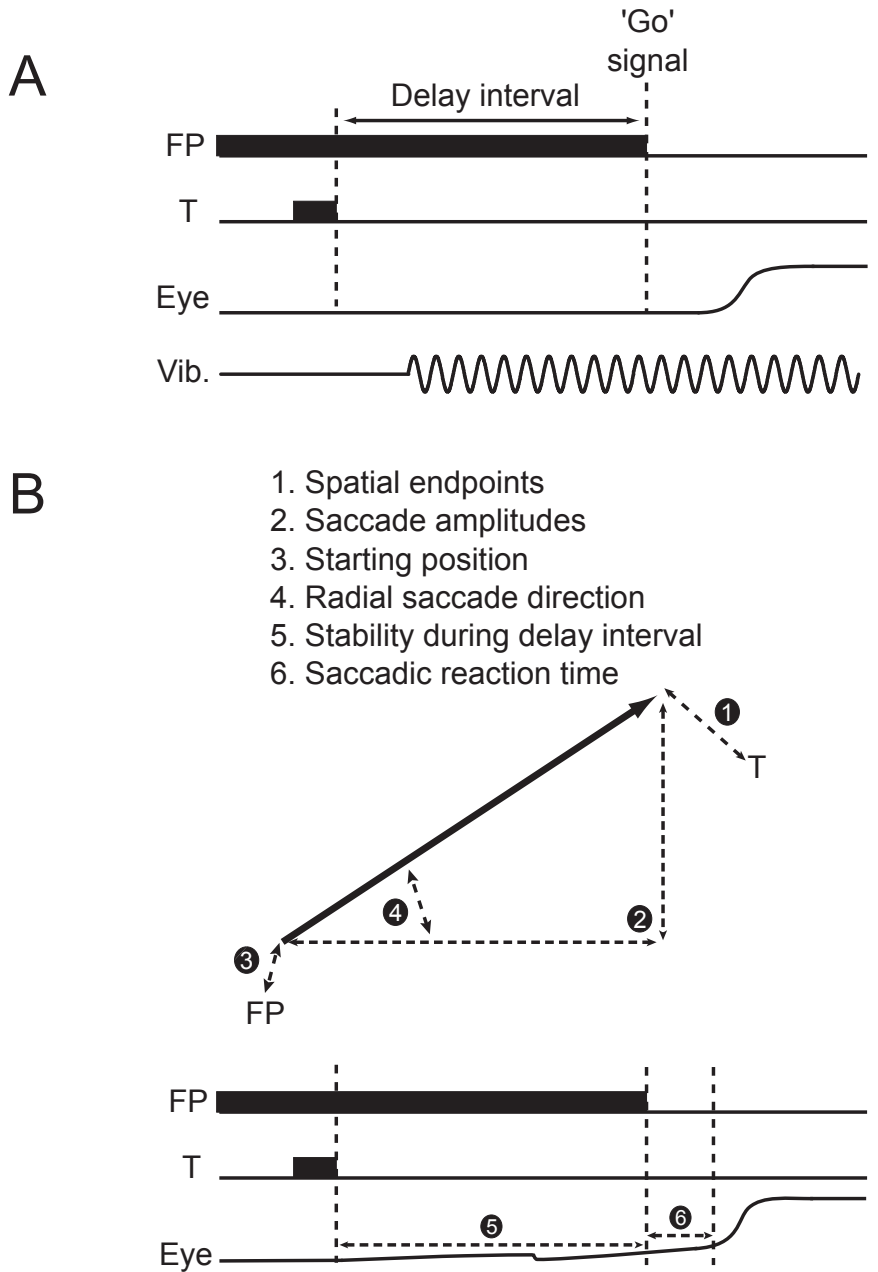


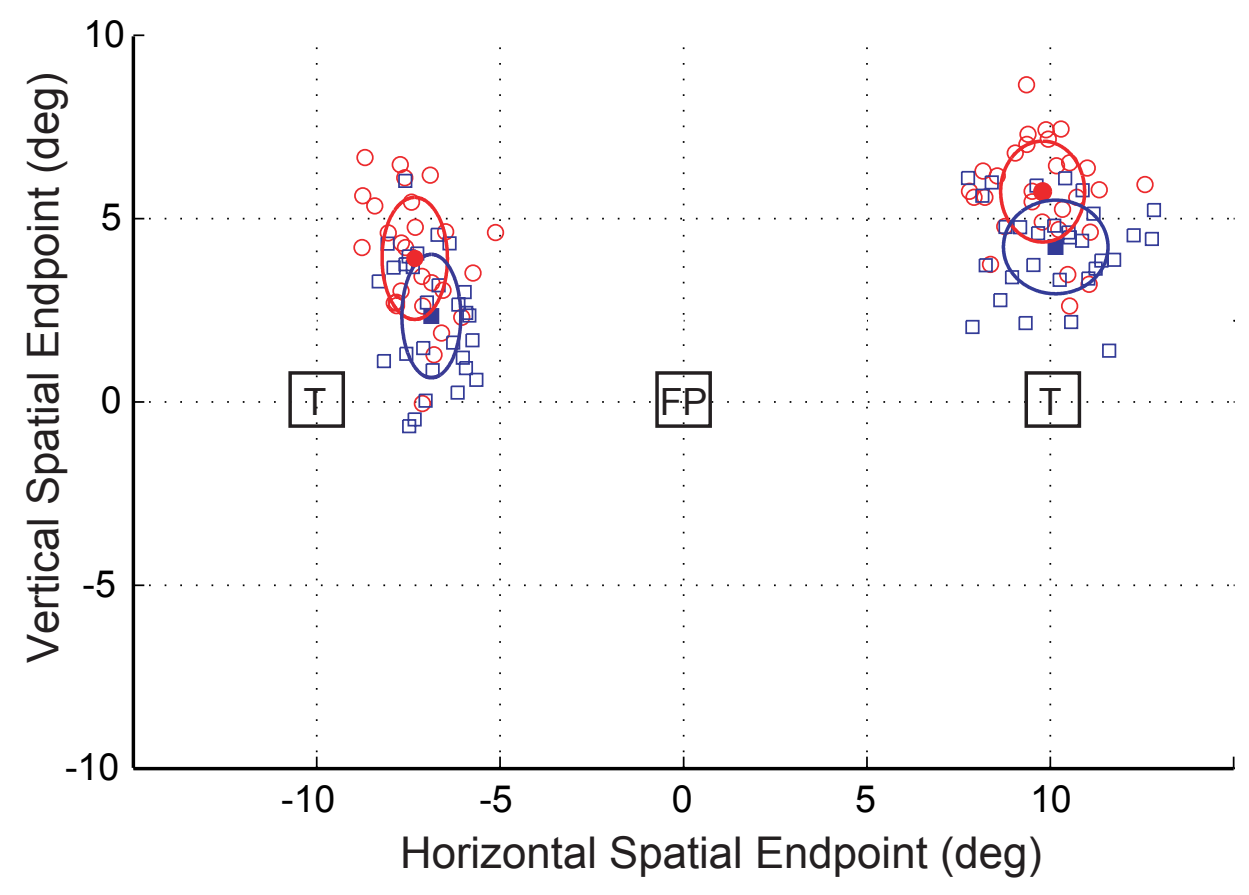

Corneil and Andersen 2004 - Fig. 2 


\section{Endpoint differences}

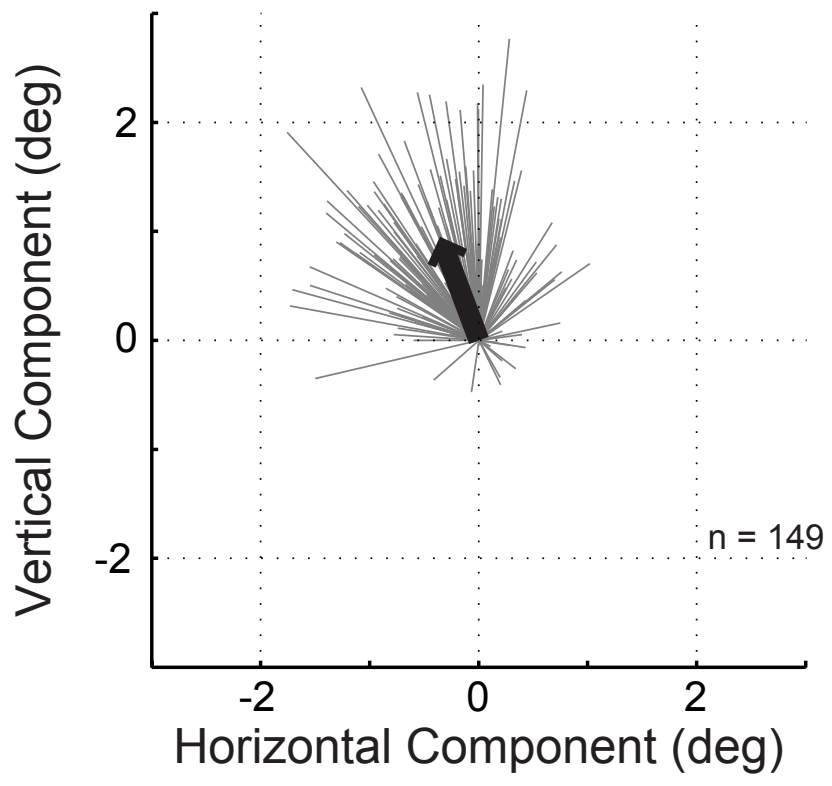

Corneil and Andersen 2004 - Fig. 3 

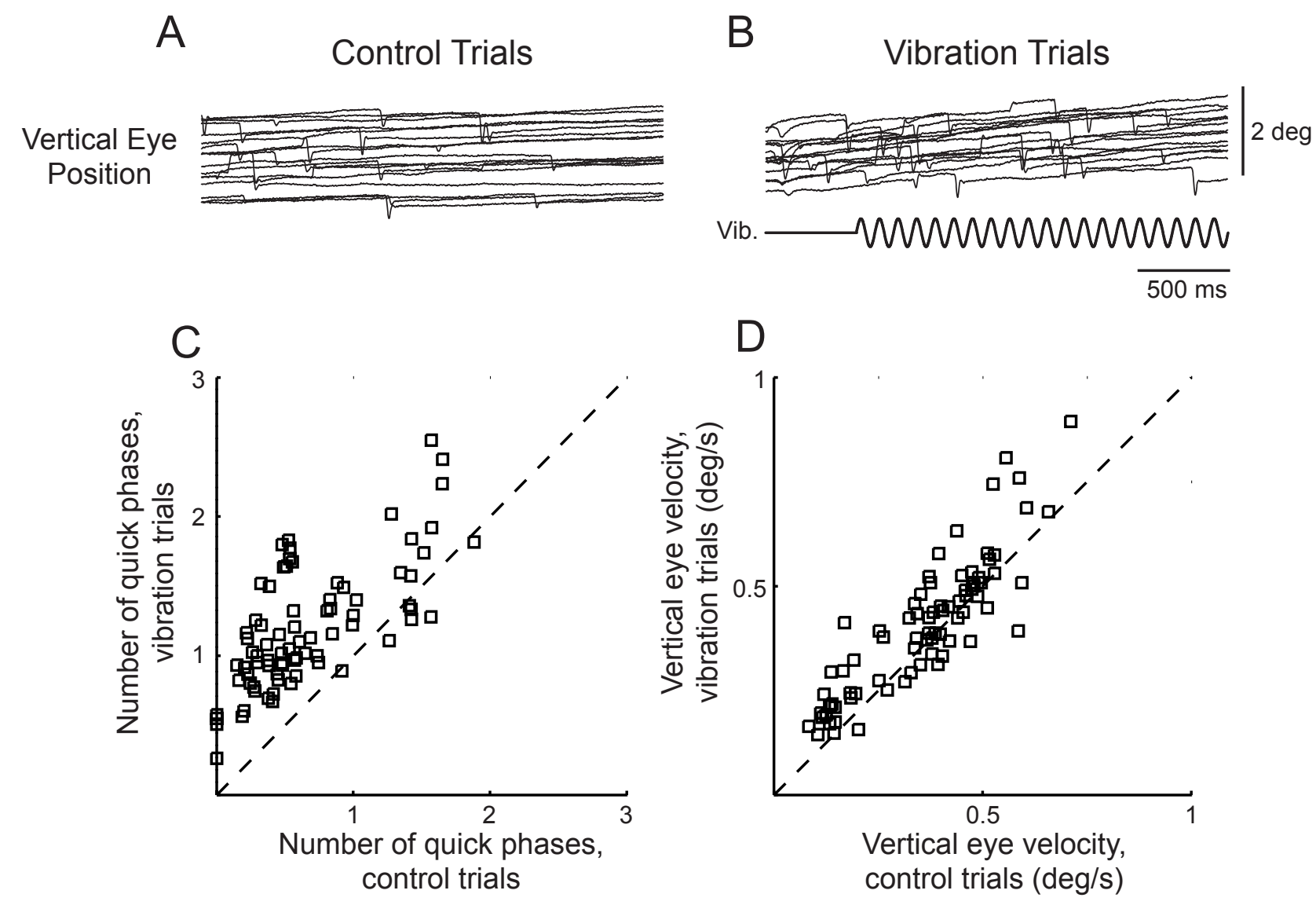

Corneil and Andersen 2004 - Fig. 4 


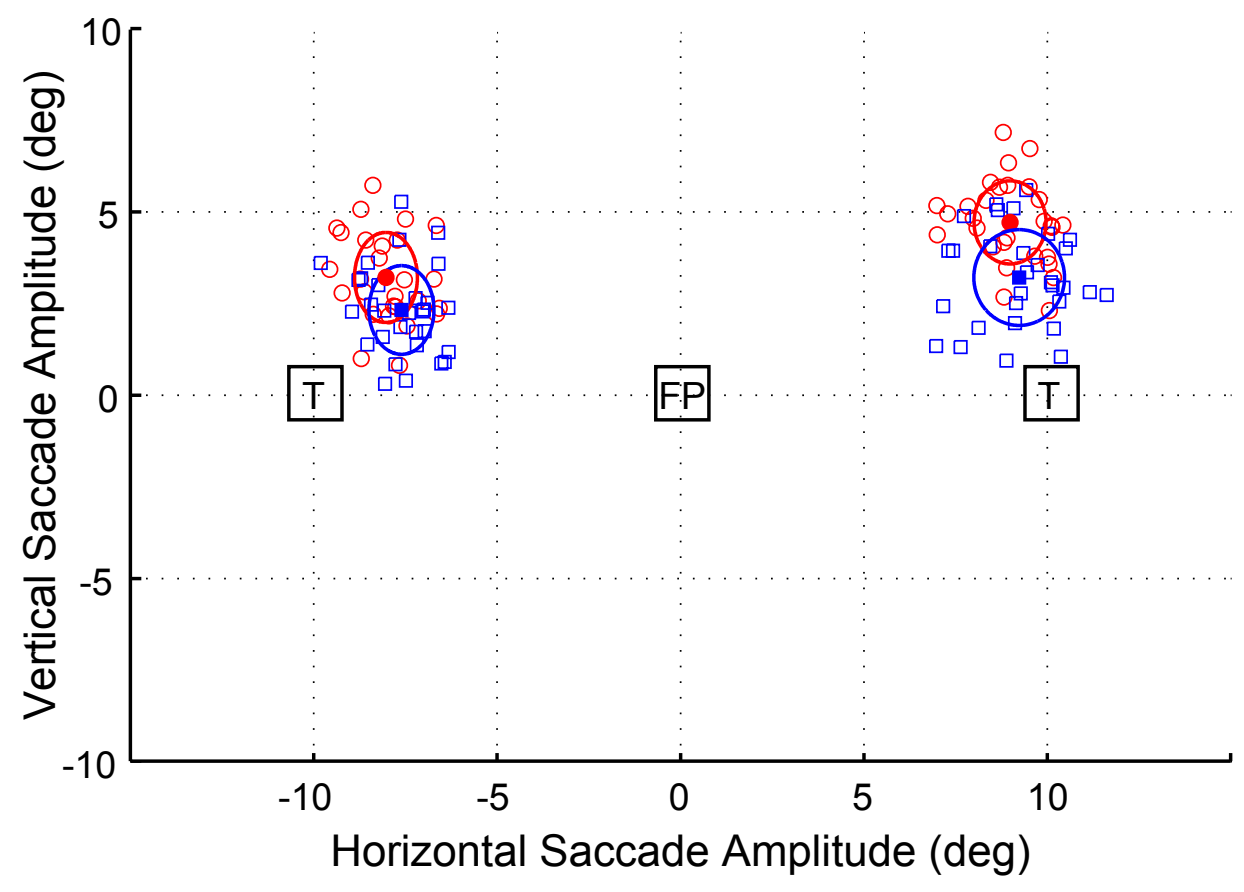

Corneil and Andersen 2004 - Fig. 5 
Saccade amplitude differences

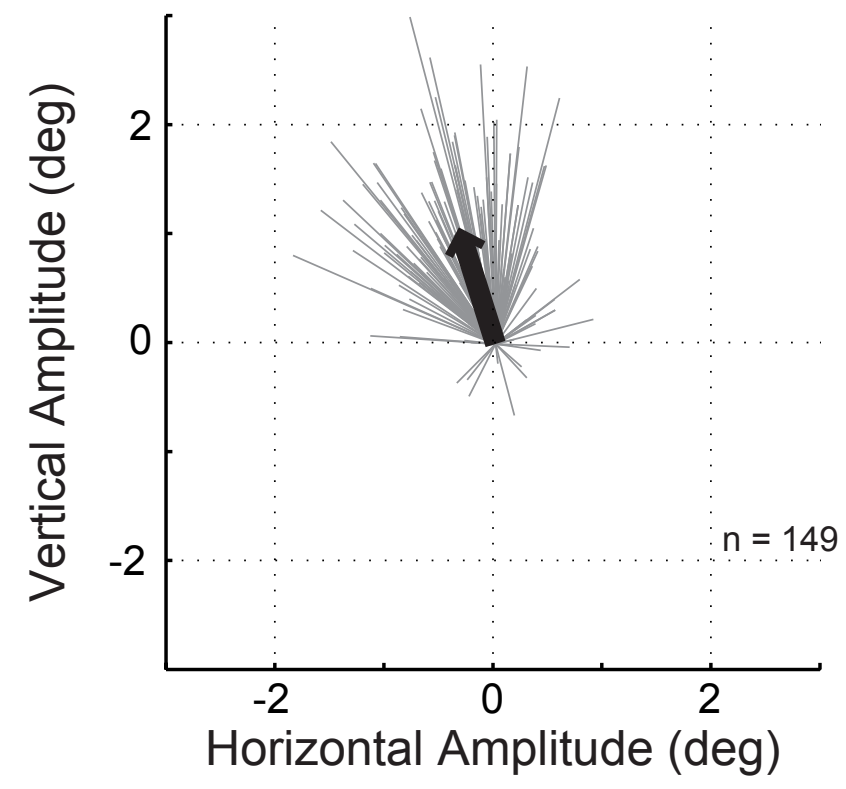

Corneil and Andersen 2004 - Fig. 6 
Saccade direction differences (deg)

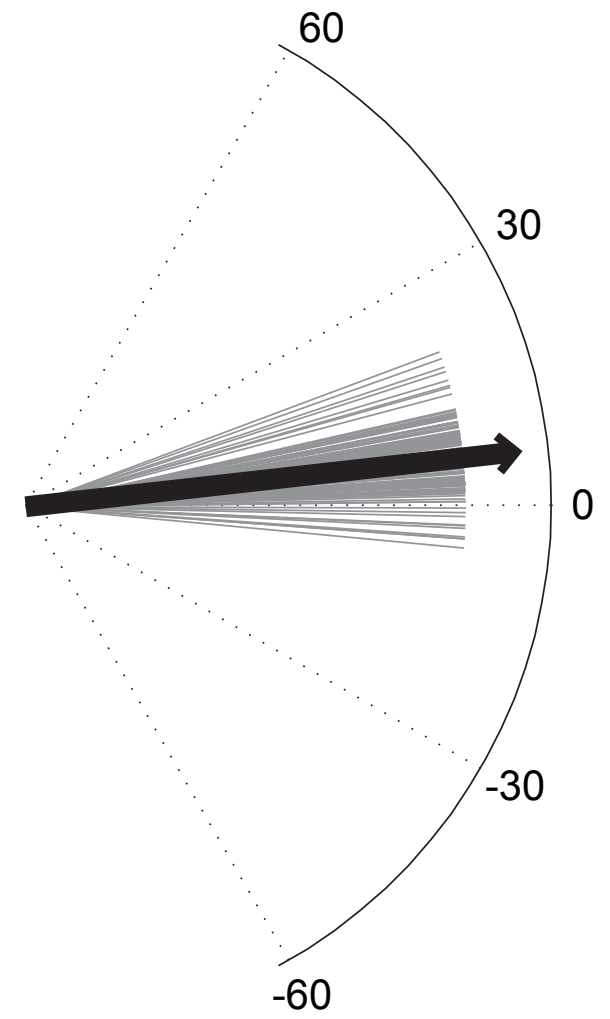

Corneil and Andersen 2004 - Fig. 7 

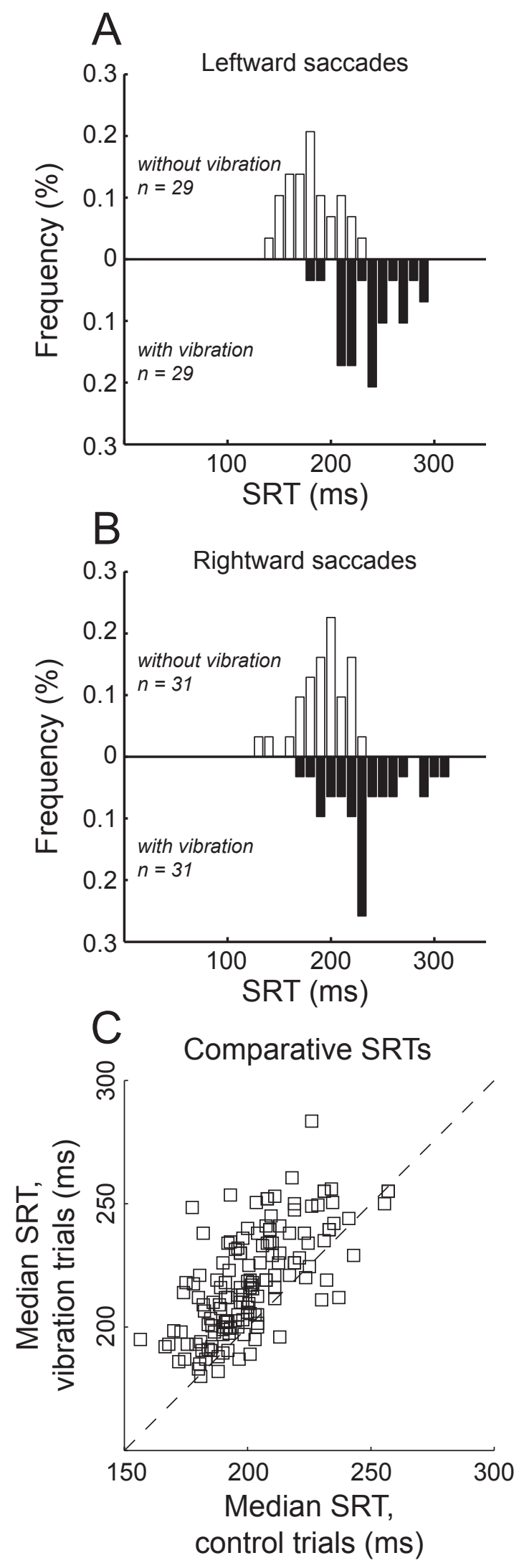

Corneil and Andersen 2004 - Fig. 8 


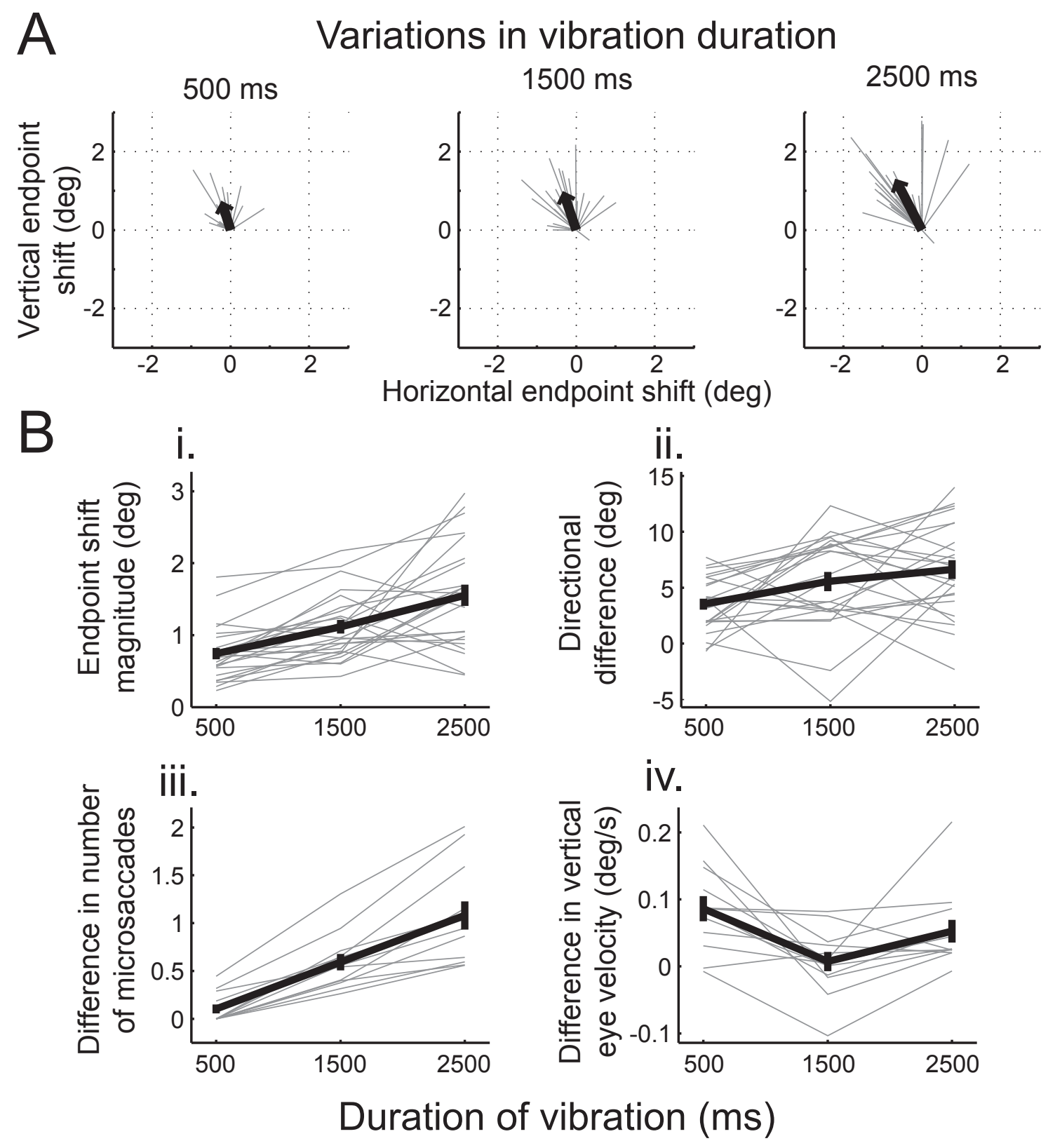

Corneil and Andersen 2004 - Fig. 9 


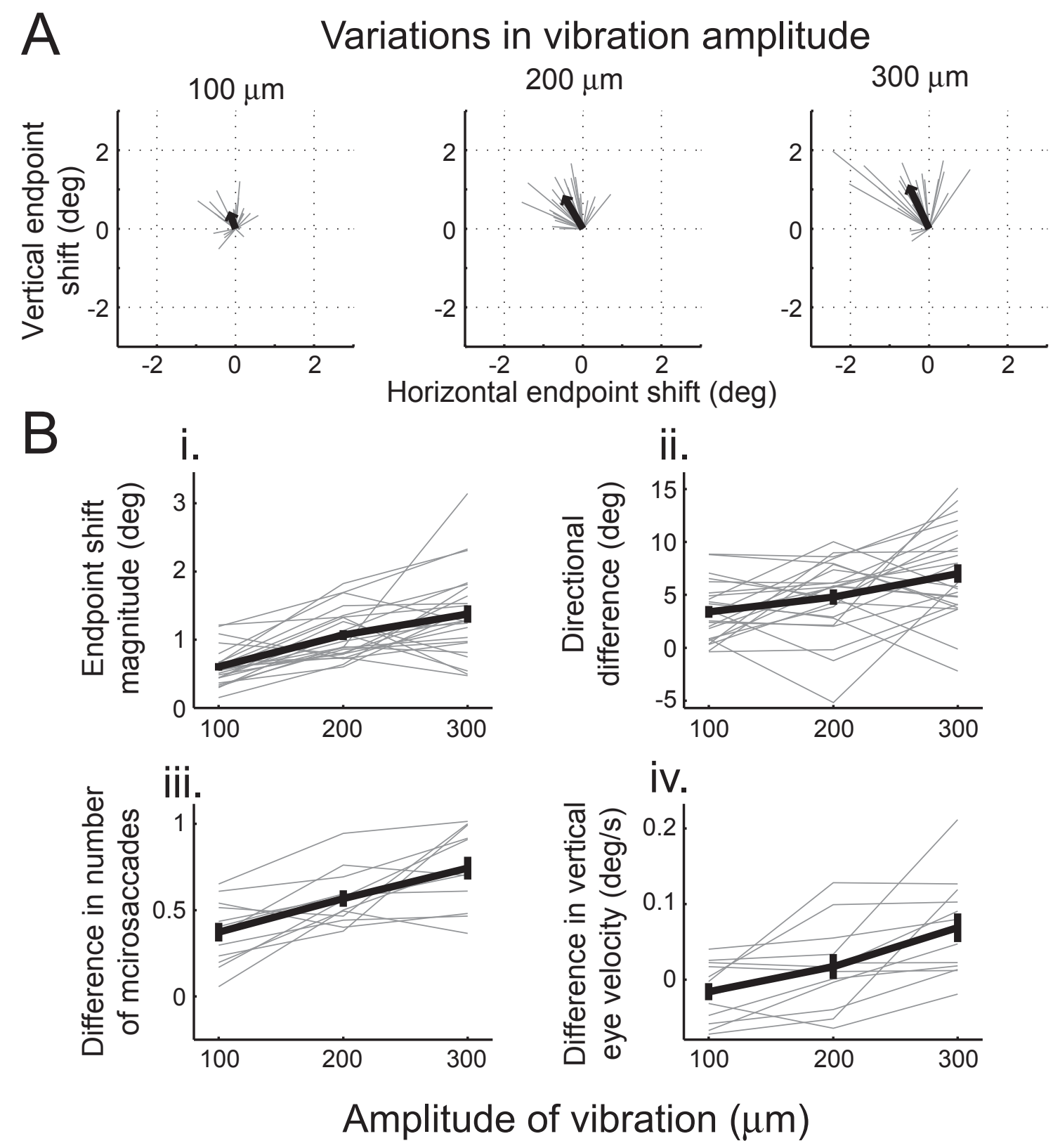

Corneil and Andersen 2004 - Fig. 10 


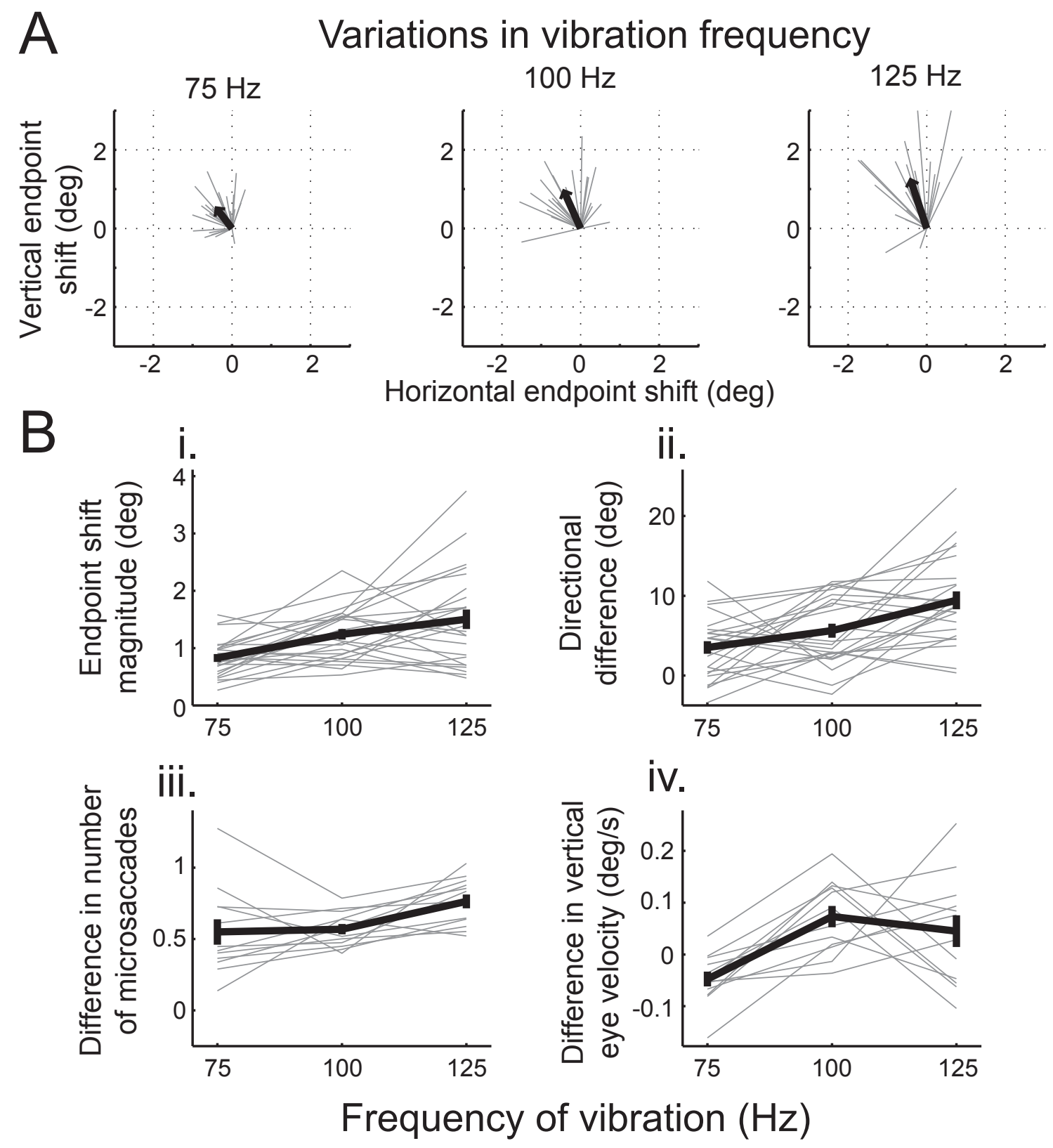

Corneil and Andersen 2004 - Fig. 11 

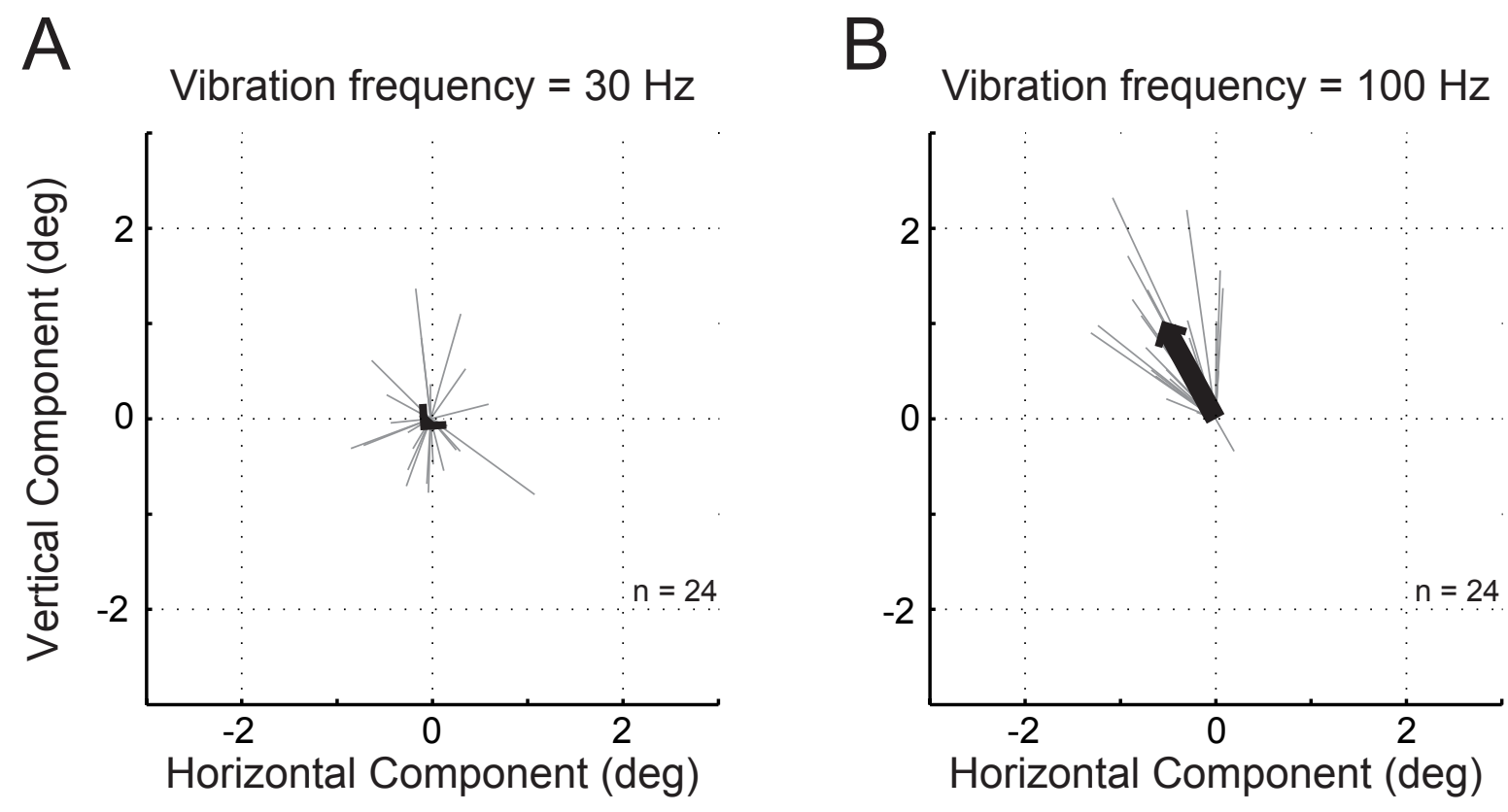

Corneil and Andersen 2004 - Fig. 12 